OPEN ACCESS

Edited by:

Renukaradhya J. Gourapura,

The Ohio State University,

United States

Reviewed by:

Loïc Deblais,

The Ohio State University,

United States

Dennis Lanning,

Loyola University Chicago,

United States

*Correspondence:

Xianghong Ju

juxh77@163.com

${ }^{\dagger}$ These authors have contributed equally to this work

Specialty section: This article was submitted to

Comparative Immunology,

a section of the journal

Frontiers in Immunology

Received: 01 June 2021 Accepted: 04 October 2021 Published: 21 October 2021

Citation:

Hu C, Niu X, Chen S, Wen J, Bao M, Mohyuddin SG, Yong $Y$, Liu X, Wu L, Yu Z, Ma X and Ju X (2021) A Comprehensive Analysis of the Colonic Flora Diversity, Short

Chain Fatty Acid Metabolism,

Transcripts, and Biochemical Indexes in Heat-Stressed Pigs.

Front. Immunol. 12:717723. doi: 10.3389/fimmu.2021.717723

\section{A Comprehensive Analysis of the Colonic Flora Diversity, Short Chain Fatty Acid Metabolism, Transcripts, and Biochemical Indexes in Heat-Stressed Pigs}

\author{
Canying $\mathrm{Hu}^{1,2 t}$, Xueting $\mathrm{Niu}^{1,2 t}$, Shengwei Chen ${ }^{1}$, Jiaying Wen ${ }^{1}$, Minglong Bao ${ }^{1}$, \\ Sahar Ghulam Mohyuddin ${ }^{1}$, Yanhong Yong ${ }^{3}$, Xiaoxi Liu ${ }^{3}$, Lianyun $W^{1}{ }^{1}$, Zhichao $\mathrm{Yu}^{3}$, \\ Xinbin $\mathrm{Ma}^{3}$ and Xianghong $\mathrm{Ju}^{1,2,3^{*}}$ \\ ${ }^{1}$ Department of Animal Science, College of Coastal Agricultural Sciences, Guangdong Ocean University, Zhaniiang, China,
${ }^{2}$ Shenzhen Institute of Guangdong Ocean University, Shenzhen, China, ${ }^{3}$ Department of Veterinary Medicine, College of
Coastal Agricultural Sciences, Guangdong Ocean University, Zhanjiang, China
}

Heat stressed pigs show typical characteristics of inflammatory bowel disease (IBD). However, little is known about the pathogenesis of heat stress (HS)-induced IBD in pigs. In this study, we determined the effects of HS on colon morphology, intestinal microbiota diversity, transcriptome genes (transcripts), and short chain fatty acids (SCFAs) metabolism in pigs. In addition, the correlation among these parameters was analyzed by weighted gene coexpression network analysis. Results showed that the liver and kidney functions related to blood biochemical indexes were partially changed in pigs under HS. Furthermore, the levels of diamine oxidase and D-lactic acid were significantly increased, whereas the levels of secretory immunoglobulin A were decreased. The integrity of colonic tissue was damaged under HS, as bleeding, lymphatic infiltration, and villi injury were observed. The concentrations of SCFAs in the colon, such as acetic acid and butyric acid, were decreased significantly. In addition, the composition of colon microbiota, such as decrease in Lactobacillus johnsonii, Lactobacillus reuteri and increase in Clostridium sensu stricto 1 of day 7 and 14 while under HS. These changes were associated with changes in the concentration of SCFAs and biochemical indexes above mentioned. Differentially expressed genes were enriched in the nucleotidebinding oligomerization domain-like receptor signaling pathway, Th17 cell differentiation, and IBD pathway, which were also associated with the changes in SCFAs. Thus, the structure, diversity of intestinal microorganisms, and changes in the levels of SCFAs in colon of heat stressed pigs changed significantly, contributing to the activation of immune response and inflammatory signal pathways and causing abnormal physiological and biochemical indexes and intestinal mucosal damage. These results highlight the interconnections between intestinal microbiota, SCFAs, and immune response and their role in the pathogenesis of stress induced IBD therapy.

Keywords: heat stress, inflammatory bowel disease, multi-omics, microbiome, short chain fatty acids 


\section{INTRODUCTION}

Heat stress (HS) can cause immunosuppression, intestinal barrier damage, and inflammation in animals that not only affect the digestion and absorption of nutrients and reduce feed reward $(1,2)$, but also increase the sensitivity of animals to a variety of infectious diseases (3). The heat-neutral range, also called the thermal comfort zone, that the appropriate ambient temperature range when basic heat and heat dissipation of body are balanced, metabolic and physiological functions of animals are at their best, of finishing pigs is $15 \sim 22^{\circ} \mathrm{C}(4,5)$. In pigs, HS is characterized by intestinal ulcers, mucosal barrier imbalance, and inflammatory cell infiltration $(6,7)$, which are the typical characteristics of inflammatory bowel disease (IBD). IBD is an inflammatory reaction caused by an imbalance between the immune system and intestinal microecosystem, induced by environmental factors on the basis of susceptible genes (8).

The systemic manifestations of HS are marked by changes in the blood, liver, kidney, and gastrointestinal tract (9-12). Cui et al. found that HS can induce oxidative stress reaction, immune response, and apoptosis of liver cells in pigs, resulting in the damage of liver function $(13,14)$. Furthermore, oxidative stress caused by HS has been shown to damage mitochondria, cell membrane and protein, and consequently affect the liver lipid metabolism and animal production performance (9). In addition, HS affects the glomerular filtration and urine concentration function of the kidneys by inducing changes in the levels of body electrolytes, especially the balance of sodium, potassium, calcium, and chloride ions, and creatinine metabolism $(15,16)$.

Several microorganisms, including bacteria, fungi, and viruses inhabit the animal intestines. The ecological community formed by these microorganisms is called intestinal microbiota, which plays an important role in digestion, metabolism, and immune regulation (17-19). When the intestinal flora is imbalanced, the intestinal epithelial cell barrier can be destroyed by the bacterial protease and various toxins produced by the intestinal microbiota (20). Leslie et al. reported that HS led to changes in the intestinal microflora structure and caused diarrhea in pigs (21), but the causal relationship between them was not completely revealed.

Short chain fatty acids (SCFAs) are metabolites of the intestinal microbiota, which are not only the energy source of the host, but also play an important role in regulating mucosal immunity by enhancing mucus secretion and promoting the development of

Abbreviations: ALT, Alanine Aminotransferase; ALB, Albumin; ALP, Alkaline Phosphatase; AMY, Amylase; BUN, Blood Urea Nitrogen; BSA, Bovine Serum Albumin; $\mathrm{Ca}^{2+}$, Calcium Ion; CCA, Canonical Correspondence analysis; $\mathrm{Cl}^{-}$, Chloride; CHOL, Cholesterol; GLO, Globulin; GLU, Glucose; HS, Heat Stress; HE, Hematoxylin-Eosin Staining; IBD, Inflammatory bowel disease; IL, Interleukin; IEC, Intestinal Epithelium Cells; KEGG, Kyoto Encyclopedia of Genes and Genomes; LAC, Lactic Acid; LEfSe, line discriminant analysis effect size; NF- $\kappa B$, Nuclear Factor $\kappa$ Appa B; OTU, Operational taxonomic unit; PAS, Periodic Acid-Schiff Stain; PBS, Phosphate buffer saline; K+, Potassium Ion; PCA, Principal Component Analysis ; PCoA, Principal Co-ordinates Analysis; RDA, Redundancy analysis; sIgA, Secretory Immunoglobulin A; SCFAs, Short Chain Fatty Acids; $\mathrm{Na}^{+}$, Sodium Ion; SPF, Specific Pathogen Free; TBIL, Total Bilirubin; tCO2, Total Carbon Dioxide; TP, Total Protein; WGCNA, Weighted Gene CoExpression Network Analysis. regulatory $\mathrm{T}$ cells $(22,23)$. It has been reported that SCFAs are involved in the maintenance of intestinal function, glucose homeostasis, and appetite and regulation of energy metabolism, inflammation, and immunity and observed in cases of tumors and colon cancer (24-26). At the same time, SCFAs are also related to the host's gut microbial composition $(27,28)$. Studies have shown that intestinal microorganisms and their metabolites are involved in the regulation of stress induced $\operatorname{IBD}(29,30)$. Till date, the correlation between intestinal microbiota, SCFAs, and immune response is not fully clear and their role in the pathogenesis of stress induced IBD is unknown.

Here we hypothesize that colonic microbiota and their metabolites may have a potential regulatory relationship with intestinal inflammation under HS. Therefore, in this study, we determined the morphological changes in colon, structure of intestinal microbiota, changes in transcriptome genes (transcripts) and SCFAs metabolism in pigs under heat-neutral range temperature and HS. In addition, the correlation among them was analyzed by weighted gene co-expression network analysis (WGCNA).

\section{MATERIALS AND METHODS}

\section{Animals and Management}

Total 48 pigs (Luchuan sows $\times$ Duroc boars; males), 2-monthold $(16 \pm 1 \mathrm{~kg})$ were housed in two animal rooms at the Animal Hospital of Guangdong Ocean University, Zhanjiang, China. Pigs with similar body weights were divided into five groups as follows: five pigs each in the day 1, 7, 14 and 21 of control groups and HS treatment groups. There were six replicates (one for each sampling date), with three pigs per group. The animals were maintained for 2 weeks at $20 \pm 2^{\circ} \mathrm{C}, 75-85 \%$ relative humidity, and $12 \mathrm{~h} / 12 \mathrm{~h}$ light/dark photoperiod to acclimatize them to the environment.

Groups for experimental animals

\begin{tabular}{lcc}
\hline Days of treament & \multicolumn{2}{c}{ Group name } \\
\cline { 2 - 3 } & Control & HS \\
\hline 1 & 6 & 6 \\
7 & 6 & 6 \\
14 & 6 & 6 \\
21 & 6 & 6 \\
\hline
\end{tabular}

Throughout the study, the pigs in each group were served a complete feed formula (Charoen Pokphand Group, China) in the morning, afternoon, and evening in $0.4 \mathrm{~kg} /$ serving with approximately 6 -h intervals between feedings. Drinking water was freely available. To minimize acute HS, the animal facility was gradually warmed $\left(2^{\circ} \mathrm{C} / \mathrm{d}\right)$ over a 7 -day period. The adaptive feeding continued for 14 days (including 7 days of gradually warming). The HS trial lasted 21 days. The control pigs were exposed to $22 \pm 3^{\circ} \mathrm{C}$, and the HS-pigs to $34 \pm 1^{\circ} \mathrm{C}$, at $75-85 \%$ relative humidity and $12 \mathrm{~h} / 12 \mathrm{~h}$ photoperiod. 
The experimental protocols for management and care of pigs were approved by the Animal Care and Use Committee of Guangdong Ocean University, Zhanjiang, China (Permit No. 206-1108).

\section{Sample Collection}

About 5-10 mL of the peripheral blood was collected from each pig through the anterior vena cava. After natural tilt coagulation for $30 \mathrm{~min}$, the blood was centrifuged $(3000 \times \mathrm{g})$ at $4^{\circ} \mathrm{C}$ for 15 min. Then, the serum was separated and sub packed and stored at $-80^{\circ} \mathrm{C}$. Subsequently, the pigs were sacrificed by electric shock fainting and bloodletting, and each segment of the colonic tissue was separated by abdominal operation. The sampling position was as consistent as possible. After the separation of adipose and mesentery of colonic tissue, the colonic tissues of each segment were cut and $10 \mathrm{~g}$ of the colon content was collected and stored at $-80^{\circ} \mathrm{C}$. Next, $1-\mathrm{cm}$ segments of the colon were cut and soaked in $10 \%$ formaldehyde solution for fixation. The other tissues were cleaned with sterile phosphate-buffered saline (PBS), cut into small pieces, packed in labeled sample bags, and quickly frozen in liquid nitrogen. After collecting, the tissue samples and intestinal contents were transferred to $-80^{\circ} \mathrm{C}$ from liquid nitrogen for cryopreservation.

During the multi-omics test, 3 samples of colonic contents were randomly taken from each group for microbiome experiments sequencing and analysis firstly. Then take the day 1 of heat-neutral range temperature treatment as the control group, analyzed the difference of the biochemical indexes of peripheral blood serum, SCFAs metabolism of colonic contents, and transcriptome of colonic tissues $(n=3)$ in day $1,7,14$ and 21 of HS treatment. Finally, analyze the correlation between different indicators of the same pig from each experimental group.

\section{Determination of Serum Biochemical Indexes}

After thawing and centrifugation $\left(3000 \times \mathrm{g}\right.$ at $4^{\circ} \mathrm{C}$ for $\left.15 \mathrm{~min}\right)$, $200 \mu \mathrm{L}$ of serum was added to the biochemical analysis systems (Tianjin Mnc Technologies, China), and serum glucose, serum cholesterol, liver function (including albumin, globulin, alanine aminotransferase, and alkaline phosphatase), the renal function (including sodium ion, calcium ion, calcium sodium ratio, chloride, creatinine), creatine kinase, total carbon dioxide, total protein, lactate, inorganic phosphorus, and amylase were determined and analyzed according to the instruction manual (Tianjin Mnc Technologies, China).

\section{Enzyme-Linked Immunosorbent Assay}

Cultural supernatant was stored at $-80^{\circ} \mathrm{C}$ prior to analysis. Serum biochemical markers were measured using ELISA kits for diamine oxidase (MeiMianBio, Wuhan, China; Cat \# MM043801), Dlactic acid (MeimianBio; Cat \# MM3373201), and secretory immunoglobulin A (sIgA MeimianBio; Cat \# MM3623401), according to the manufacturer's protocol. The intact supernatant was used in the following analysis. The plates were read using a microplate reader (BioTek Instruments Inc., Winooski, VT, USA) at a wavelength of $450 \mathrm{~nm}$. A standard curve for each of the cytokines was used to estimate the concentration. SPSS 19.0 (SPSS, Chicago, Illinois) was used for statistical analysis. The differences among the groups were analyzed by one-way ANOVA, student-T test was used for multiple comparisons. The difference was considered statistically significant for $P<0.05$.

\section{Morphological Observations}

For histopathological examination, the colonic tissue was fixed in buffered formalin $(10 \% \mathrm{v} / \mathrm{v})$ and stained with hematoxylin and eosin. Image-Pro Plus v. 6.0 (Media Cybernetics Inc., Silver Spring, MD, USA) was used to measure villus height, crypt depth, and width (1). Hydrated colonic tissue sections were treated with amylase at $37^{\circ} \mathrm{C}$ for $1 \mathrm{~h}$, rinsed under running water for $10 \mathrm{~min}$, and stained with periodic acid solution at room temperature $\left(25 \pm 2^{\circ} \mathrm{C}\right)$ for $7 \mathrm{~min}$, according to the instructions for the Glycogen D-PAS Staining Kit (Leagene Biotechnology, Beijing, China). The tissue sections were rinsed with tap water, immersed in Schiff s reagent in the dark for $15 \mathrm{~min}$, and rinsed with tap water for $10 \mathrm{~min}$ to remove the stain. The sections were dehydrated with an alcohol concentration gradient $(75 \%, 85 \%$, $95 \%$, and $100 \%$ ), cleared of alcohol with xylene, and sealed with neutral gum. Image-Pro Plus v. 6.0 (Media Cybernetics Inc.) was used to evaluate the goblet cells per unit area in the colonic mucosa (31).

\section{Immunofluorescence}

Paraffin sections were baked at $55^{\circ} \mathrm{C}$ and dewaxed thoroughly in xylene, then soaked in gradient alcohol and washed with PBS. The repaired tissues were treated with sodium citrate repair solution at $92^{\circ} \mathrm{C}$ for $10 \mathrm{~min}$ and blocked with $10 \%$ fetal bovine serum for $30 \mathrm{~min}$. IgA (1:500, ABclonal Technology Co., Ltd., China, Cat \# MM3373201) primary antibody was incubated for $12 \mathrm{~h}$ in $4^{\circ} \mathrm{C}$, and goat anti rabbit $\operatorname{IgG}(\mathrm{H}+\mathrm{L})$ PE fluorescent labeled second antibody (1:500, TransGen Biotech Co., Ltd, China, Cat \# HS121-01) was incubated for $2 \mathrm{~h}$ in $4^{\circ} \mathrm{C}$, DAPI was dripped after three times washing with PBS, and the stained tissue was observed and photographed under the microscope system (Cat \#IX73, Olympus Corporation, Japan).

\section{Determination and Analysis of SCFAs Concentration in the Colonic Content}

Fifty microliters of $15 \%$ phosphoric acid were added to $50 \mathrm{mg}$ of colonic content, then $100 \mu \mathrm{L}$ of $125 \mu \mathrm{g} / \mathrm{ml}$ internal standard (isohexanoic acid) solution and $400 \mu \mathrm{L}$ ether were added, tissue homogenate for $1 \mathrm{~min}$, then centrifugation at $4^{\circ} \mathrm{C}$ and $12000 \times \mathrm{g}$ for $10 \mathrm{~min}$. The supernatant was analyzed by gas chromatography-mass spectrometry (GC-MS, Agilent Technologies Inc., Agilent 6890N/5975B, USA). The column used was Agilent HP innowax capillary column $(30 \mathrm{~m} \times 0.25 \mathrm{~mm}$ ID $\times 0.25 \mu \mathrm{m})$ in the split injection mode. The injection volume was $1 \mu \mathrm{L}$ and split ratio was 10:1. The temperatures of the injection port, ion source, line, and quadrupole were $250^{\circ} \mathrm{C}$, $230^{\circ} \mathrm{C}, 250^{\circ} \mathrm{C}$, and $150^{\circ} \mathrm{C}$, respectively. The initial temperature of the programming was $90^{\circ} \mathrm{C}$. The temperature was raised to $120^{\circ} \mathrm{C}$ (at $10^{\circ} \mathrm{C} / \mathrm{min}$ ), followed by an increase to $150^{\circ} \mathrm{C}$ (at $5^{\circ}$ $\mathrm{C} / \mathrm{min}$ ). Finally, the temperature was raised to $250^{\circ} \mathrm{C}$ for $2 \mathrm{~min}$ (at $25^{\circ} \mathrm{C} / \mathrm{min}$ ). The carrier gas was helium with a flow rate of 
$1.0 \mathrm{~mL} / \mathrm{min}$. The mass spectrometry used an electron impact ionization source and SIM scanning mode, and the electron energy was $70 \mathrm{ev}$ (Data Sheet 1).

Based on the detection results, targeted quantification was carried out for the detected samples, and the relevant data were analyzed according to the quantitative results. According to the established sample pretreatment and instrumental analysis methods and standards, all samples were analyzed quantitatively. PCA was used to generate new characteristic variables by linear combination of metabolite variables according to a certain weight. The samples with poor repeatability (outlier samples) and abnormal samples were removed. The results of the orthogonal projections to latent structures discriminant analysis (OPLS-DA) can effectively reduce the complexity of the model and enhance the explanatory ability of the model without reducing the predictive ability of the model, so as to maximize the difference between groups. On this basis, the differences in metabolites between groups were analyzed to the maximum extent by using OPLS-DA.

SPSS19.0 was used to calculate the mean value and standard deviation of each group, and calculate the statistically significant $\mathrm{P}$-value. Z-score (standard score) was calculated based on the content of metabolites, which is used to measure the content of metabolites at the same level, using the following formula: $\mathrm{z}=$ $(\mathrm{x}-\mu) / \sigma$ ( $\mathrm{x}$ is a specific fraction, $\mu$ is the average, $\sigma$ is the standard deviation. The cor-function in $\mathrm{R}$ (v3.1.3) was used to calculate the correlation coefficient. When the linear relationship between the two metabolites was enhanced, the correlation coefficient tended to 1 or -1 : positive correlation tended to 1 , negative correlation tended to - 1 . At the same time, the method of $\mathrm{R}$ is used cor.test $P \leq 0.05$ had significant correlation.

\section{Microbial Genomic Sequencing}

Total genomic DNA was extracted from the samples $(n=3)$ with a QIAamp DNA Stool Mini Kit (Qiagen, Hilden, Germany), according to the manufacturer's instructions. DNA concentration and purity were evaluated on 1\% agarose gel. The quantity of DNA was determined with a NanoDrop 1000 spectrophotometer (Thermo Fisher Scientific, USA) after calibration with sample solvents and the DNA was diluted to 1 $\mathrm{ng} / \mu \mathrm{L}$ with sterile water. The V3-V4 distinct regions of the $16 \mathrm{~S}$ rRNA genes were amplified with specific barcoded primers (32, 33). The PCR reactions were performed in triplicate in a total volume of $25 \mu \mathrm{L}$, consisting of $1 \mu \mathrm{L}$ of each the primers $(5 \mu \mathrm{M})$, $10 \mu \mathrm{L}$ of $10 \mathrm{ng}$ DNA template, $4 \mu \mathrm{L}$ of $1 \times$ FastPfu buffer, $1 \mu \mathrm{L}$ of $2.5 \mathrm{mM}$ dNTPs, $0.4 \mu \mathrm{L}$ FastPfu polymerase, and $7.6 \mu \mathrm{L}$ nucleasefree water. The PCR program was as follows: initial denaturation at $94^{\circ} \mathrm{C}$ for $5 \mathrm{~min}, 30$ cycles at $94^{\circ} \mathrm{C}$ for $50 \mathrm{~s}, 55^{\circ} \mathrm{C}$ for $30 \mathrm{~s}, 72^{\circ} \mathrm{C}$ for $50 \mathrm{~s}$, and a final extension at $72^{\circ} \mathrm{C}$ for $6 \mathrm{~min}$. The PCR products were purified with an AxyPrep DNA Gel Extraction Kit (Axygen Scientific Inc., USA). Amplicons from all samples were sent to a commercial company (Biomarker Technologies Corporation, China) for sequencing on an Illumina HiSeq 2500 platform (Illumina, USA). Sequence/expression data had been uploaded to NCBI database (PRJNA729581 and PRJNA744592 for heat stressed-pigs colonic microbiome project).

\section{Microbial Genomic Analyses}

Species classification information corresponding to each operational taxonomic unit (OTU) was obtained by comparing the representative OTU sequences with the microbial reference database (SILVA, v. 138 and ITS, v 8.3). After rarefying OTU samples (RDP Classifier. 2.2; the confidence threshold is 0.8 , sample size < minimum of samples (40000 reads) (34), sample community compositions were calculated at the phylum, class, order, family, genus, and species levels and generated in QIIME 2 (v 2021.2). GraphPad Prism v. 7.0 c (GraphPad Software, USA), R (v 3.1.3), Metastats, and STAMP (Statistical Analysis of Metagenomic Profiles) were used for the statistical analyses. The weighted UniFrac distances among the groups were statistically compared by analysis of similarities using the 'vegan' package of $\mathrm{R}$ ( $\mathrm{v}$ 3.1.3). QIIME 2 software for Alpha diversity index of each sample, including the Shannon and Simpson indices. PERMANOVA/ANOSIM analysis is performed with binary jaccard (for unweightde unifrac) and bray Curtis, to cal (for weighted unifrac) culate the sample distance. We used $\mathrm{R}$ (v3.1.3) to draw rank abundance curves, species accumulation curves, PCA plots, and a heatmap of sample distances. PICRUST software was used to compare the species composition information obtained from $16 \mathrm{~S}$ sequencing data to determine the functional gene composition of the samples (35), and the functional differences between different samples or groups were analyzed. Use PICRUST to compare the species composition information obtained by comparing $16 \mathrm{~S}$ sequencing data, infer the functional gene composition in the sample, and analyze the functional differences between different samples or groups. In the univariate analysis of gut microbiota and predicted KEGG biochemical pathways for each group, a one-way ANOVA with Bonferroni's multiple comparison test was performed to compare the diversities among the groups. Metastats identified differentially abundant phyla, genera, classes, and species in the groups. Significant differences between groups were identified by the LEfSe (Line Discriminant Analysis [LDA] Effect Size) method. At the genus level, the G-TEST and Fisher test were used to detect the differences in the abundance of species between samples. Pairwise t-tests were used to detect differences between groups, assuming a P-value threshold for significance of 0.05 . RDA (Redundancy analysis) or CCA (Canonical Correspondence analysis) (36), distance-based redundancy analysis (db-RDA), and mantel test analysis in $\mathrm{R}$ language vegan package were used to analyze and map the relationships between the concentrations of SCFAs and serum biochemical indexes and changes in microbial flora.

\section{Transcriptome Sequencing and Analysis}

In order to simplify the complexity of data analysis, we removed the time effect and only considered the influence of heat stress treatment on metabolism and intestinal changes. The enzymaticfree cryopreservation tube was pre-cooled in liquid nitrogen, and the tissue was quickly removed from the living body (cut into pieces sizes similar to soybean granules). Using RNase-free water to prepare $1 \times$ PBS or saline, the tissue surface stains were quickly cleaned, and the surface liquid was absorbed and collected into 
the freezing tube. Then, the freezer tube was rapidly put into liquid nitrogen for cryopreservation, and the samples were sent to the Sequencing Company (Majorbio, China) for sequencing. Eukaryotic mRNA sequencing is based on the HiSeq platform for sequencing all mRNAs transcribed from specific tissues of eukaryotes at a particular time. The total RNA was extracted from the tissue samples, the concentration and purity of the extracted RNA were detected by Nanodrop2000, the RNA integrity was detected by agarose gel electrophoresis, and the RIN value was determined by Agilent 2100. The eukaryotic mRNA 3' end had a structure of a ployA tail, and the magnetic beads with Oligo (dT) were used for A-T base pairing with the flo $A$, and mRNA could be isolated from the total RNA for analysis of transcriptome information. The fragmentation buffer was added to randomly break the mRNA into small fragments of about $300 \mathrm{bp}$. Under the action of reverse transcriptase, six-base random hexamers were added, and mRNA was used as a template to reverse the synthesis of one-strand CDNA, followed by two-strand synthesis to form a stable double-stranded structure. After connecting to the adaptor, the short sequence fragments were sequenced using the Illumina HiSeq platform. Since the Illumina sequencing single-run can generate billions of reads, we used statistical methods to statistically quality-control the measured sequences by using Fastx toolkit ( $\mathrm{v}$ 0.0.14, minimum sequence length: 30 ; minimum quality value: 20 ), which can visually reflect the library construction quality and sequencing quality of the samples. Hisat2 (v 2.2.1) was used for sequence alignment. And the quality data after the quality control (reads) was compared with the reference genome (Sus scrofa, v 11.1, Ensembl) to obtain the mapped data (reads) for subsequent analysis, and the quality of the comparison results of the sequencing is evaluated. New transcript function were noted by DIAMOND ( $\mathrm{v}$ 0.8.37.99) for NR (NCBI non-redundant protein database) and Swiss-Prot.

Based on the existing reference genomes, the mapped reads were assembled and spliced by using software Cufflinks, compared with known transcripts, transcripts without annotation information, and functional annotations of potential new transcripts. Read Counts for each sample gene/ transcript were obtained using RSEM (37). Using alignment to genome results and genome annotation files. This was then subjected to transcripts per million reads (TPM) conversion to obtain standardized gene/transcript expression levels by DESeq2. After obtaining the number of Read Counts of the gene/ transcript, the differential analysis of the expression of the gene/transcript between the samples was performed on the multi-sample $(\geq 2)$ project, and the differentially expressed genes/transcripts were identified to study the differentially expressed genes/transcription.

Weighted gene co-expression network analysis (WGCNA) was used to calculate the correlation coefficient between any two genes. To determine whether two genes have similar expression patterns, WGCNA analysis uses the weighted value of correlation coefficient, that is, the correlation coefficient of genes is N-th power, which makes the connection between genes in the network obey scale-free networks. Use the WGCNA package (v 1.46) for weighted correlation network analysis in R (v3.1.3). The WGCNA $\mathrm{R}$ software package is a comprehensive collection of $\mathrm{R}$ functions for performing various aspects of weighted correlation network analysis. The package includes functions for network construction, module detection, gene selection, calculations of topological properties, data simulation, visualization, and interfacing with external software. The SCFAs metabolite data and serum biochemical index data were introduced, and the hierarchical clustering tree was constructed by the correlation coefficient between genes. Genes with low expression or low coefficient of variation are generally considered noise, so the data for WGCNA needs to be pre-processed as much as possible to eliminate outliers. After data preprocessing, the genes/transcripts can be classified, and the expression patterns can be divided into modules. After obtaining the module, through the correlation analysis with the phenotypic data, the key module is obtained. Obtain the hub gene of the module through visualized network analysis. Different branches of the clustering tree represent different gene modules, and different colors represent different modules. Based on the weighted correlation coefficient of genes, genes are classified according to expression patterns, and genes with similar patterns are classified into a module. According to the module analysis, the correlation with environmental factors was analyzed, and the prediction network of gene/transcript interaction was drawn by Cytoscape (v. 3.6.0).

\section{RESULTS}

\section{Effects of HS on Blood Biochemical Indexes in Pigs}

The concentrations of serum albumin and globulin gradually increased with the extension of HS compared with that in control, and showed the significance in day 14 of heat stressed pigs $(P<0.05)$ (Figures 1A, B). Alanine aminotransferase level was significantly $(P<0.01)$ higher on day 1 of $\mathrm{HS}$ than that in the control group (Figure 1C). Compared with the control group, alkaline phosphatase was significantly increased $(P<0.01)$ after HS treatment on days 1, 14, and 21 of HS, but no show the difference between the control and the day 7 of HS (Figure 1D). As shown in Figures 1E-G, there was no significant change in serum calcium and sodium after HS treatment, but the ratio of calcium and sodium decreased significantly compared with that in the control group on day 7 of HS. While compared with the control, the level of chloride ion and creatinine were decreased on day 7 of HS, but it also showing the rising trend on days 1, 14, and 21 of HS (Figures 1H, I).

Compared with the control group, the concentration of glucose was significantly increased $(P<0.01)$ on days 1 and 7 of HS (Figure 2A), while the concentration of cholesterol and total protein were significantly increased $(P<0.05)$ on day 14 of HS (Figures 2B, C). The concentration of inorganic phosphorus was significantly higher $(P<0.01)$ than that in control group on days 1, 14, and 21 of HS (Figure 2D), but no show the difference between the control and the day 7 of HS. The levels of lactate and amylase in the HS group were higher than that in the control 
A

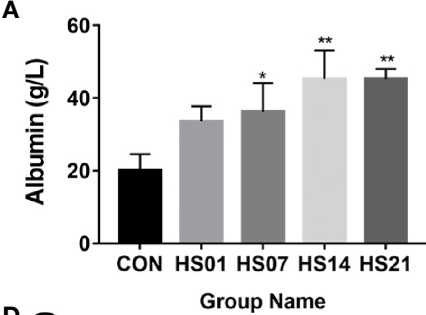

D

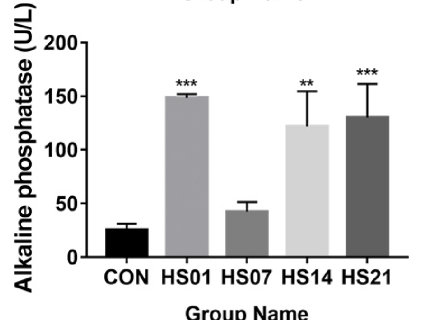

G

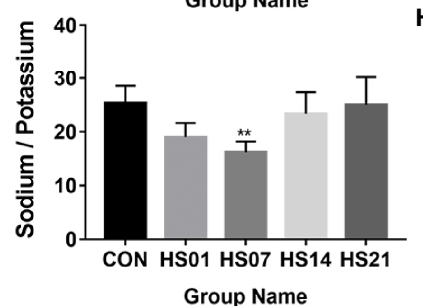

B
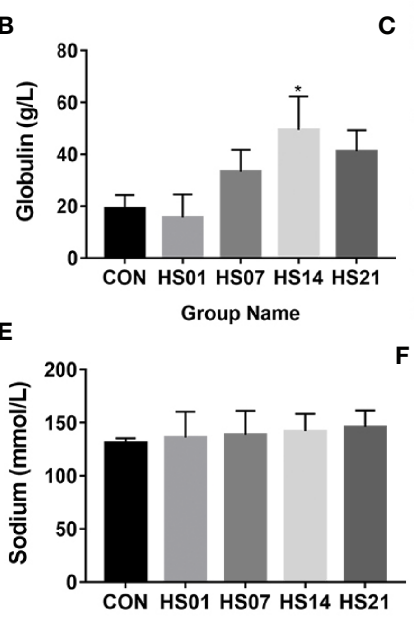

Group Name

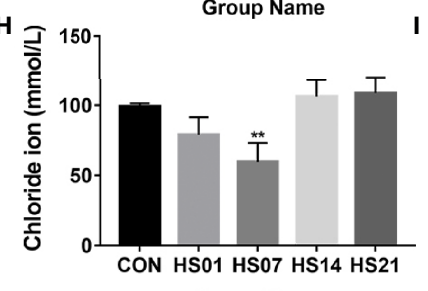

Group Name c
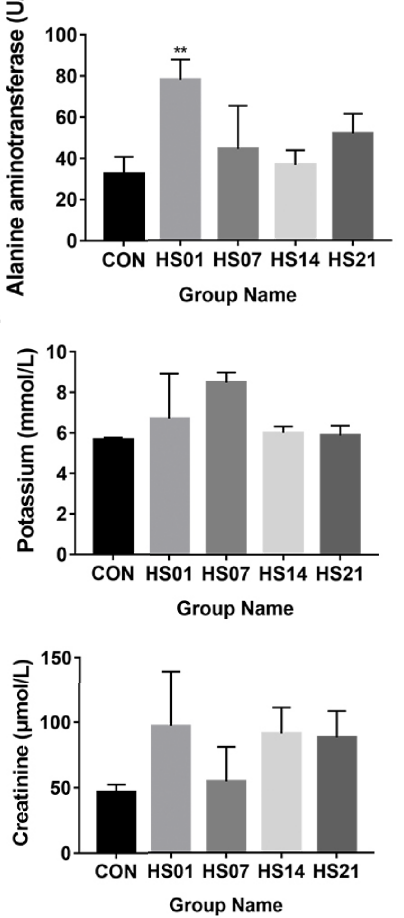

FIGURE 1 | Effect of HS on the biochemical indexes of pig serum. CON indicates no heat stress group of day 1, HS1, 7, 14, 21 indicate heat stress day 1, 7, 14,

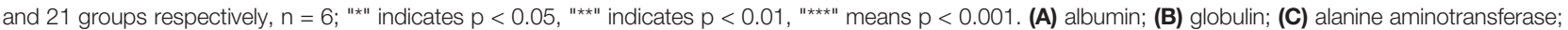
(D) alkaline phosphatase; (E) sodium ion; (F) calcium ion; (G) calcium to sodium ratio; (H) chloride ion; (I) creatinine.

group (Figures 2E, F). Compared with the control group, the total carbon dioxide was significantly decreased $(P<0.001)$ on days 1 and 7 of HS and increased significantly $(P<0.01)$ on day 14. The concentration of creatine kinase was significantly increased $(P<0.05)$ in the HS group compared with that in the control (Figures 2G, H).

The D-lactic acid was only showing the significantly $(P<0.05)$ increased on day 14 of HS, but don 't have difference on days 1,7 , and 21, by comparing with the control group (Figure $\mathbf{3 A}$ ). Compared with the control group, the concentrations of serum diamine oxidase were significantly increased on days 7 and 14 of HS $(P<0.01)$, peaked at day 14 , and decreased at day 21 (Figure 3B). Compared with the control group, HS treatment significantly reduced $(P<0.01)$ the level of Secretory immunoglobulin A ( $\operatorname{sg} \mathrm{A})$ in the colonic content (Figure 3C). And as showing in Figure 3D, the sIgA was mainly located in the intestinal epithelium and lymph nodes (as the arrow mentioned) of the colon.

\section{Effect of HS on the Morphological Structure of Pig Colon}

After HS treatment, the colonic mucosal were loosely arranged and showed signs of bleeding on days 1, 7 and 14 of HS, the local injury and lymphatic infiltration also had been found on days 7 and 14 of HS (Figure 4A). The intestinal lymph nodes were enlarged on day 14 of HS (Figure 4A). The mucosal height/crypt depth of colon was significantly decreased $(P<0.001)$ on days 1 ,
7, and 14 of HS (Figure 4B). Periodic acid-Schiff (PAS)-positive cells in the colon of HS pigs were significantly decreased $(P<$ 0.0001 ) than those in the control group (Figure 4C). The thickness of the muscle layer decreased on days 7 and 14 of HS compared with that in the control group (Figure 4D).

\section{Effect of HS on the Metabolism of SCFAs in Pig Colon}

After analysis, we found that the microbes of the control group were not significantly different at different time points. Subsequently, we used SCFAs metabolome and transcriptome tests to analyze whether microbial changes can cause the activation of intestinal inflammation pathways through SCFAs. In order to simplify the complexity of data analysis, we removed the time effect and only considered the influence of heat stress treatment on metabolism and intestinal changes. As shown in Figure 5, the concentrations of acetic acid, propionic acid, butyric acid, isobutyric acid, and caproic acid were significantly reduced $(P<0.05)$ in the HS group compared with that in the control groups, and gradually reached control levels by day 21 ; and the valeric and isovaleric acids were shown the same trend but not significant (Figures 5A-G). The metabolite variables were grouped by linear combination according to certain weight, and the data of each group were classified by main new variables (principal component analysis [PCA]), and samples with poor repeatability and abnormal samples were removed. As a result, 

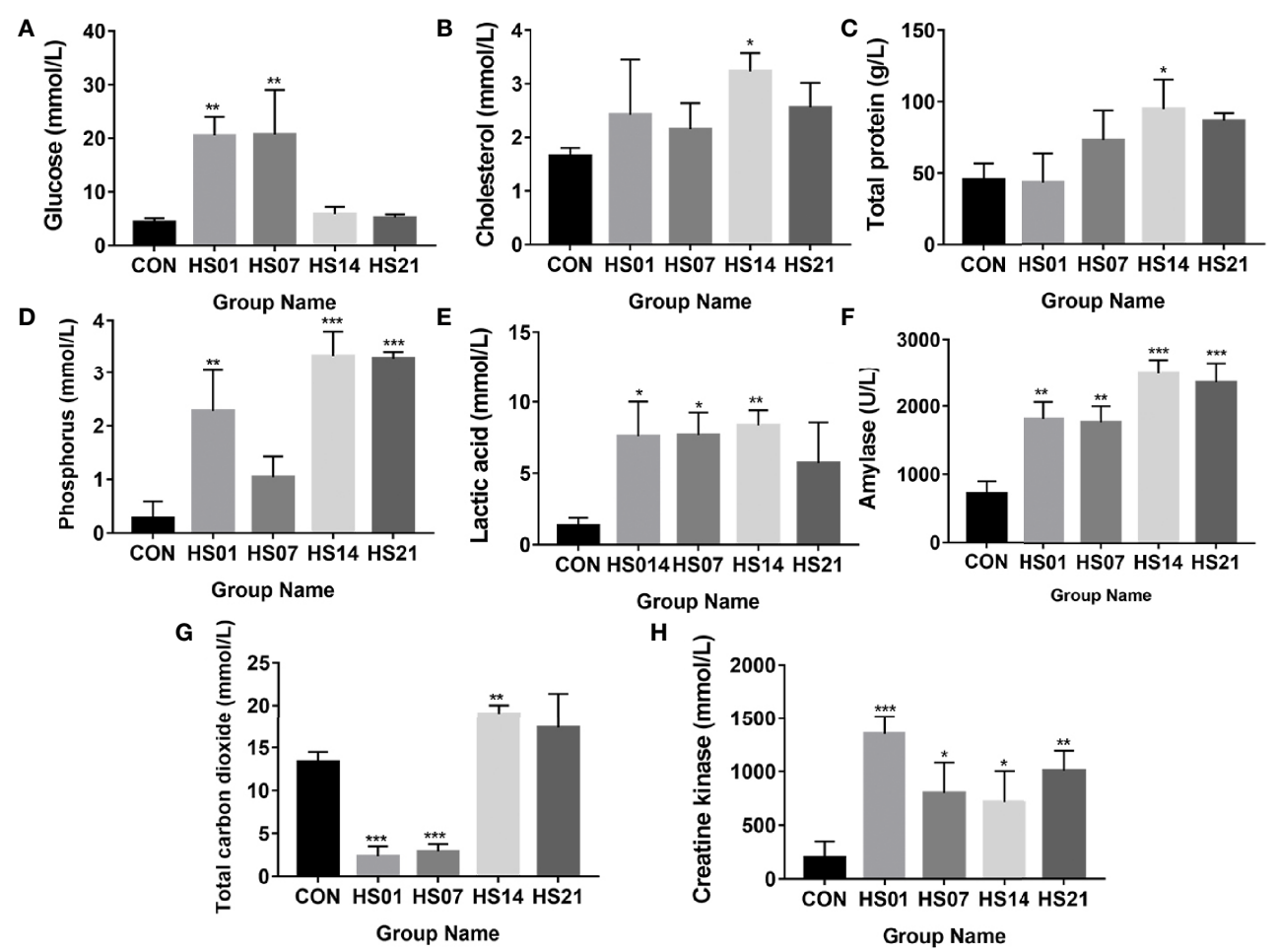

FIGURE 2 | Effect of HS on biochemical indexes in pig serum. CON indicates no heat stress group of day 1, HS1, 7, 14, 21 indicate heat stress day 1, 7, 14, and

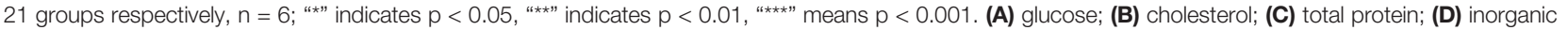
phosphorus; (E) lactic acid; (F) amylase; (G) total carbon dioxide; $(\mathbf{H})$ creatine kinase.
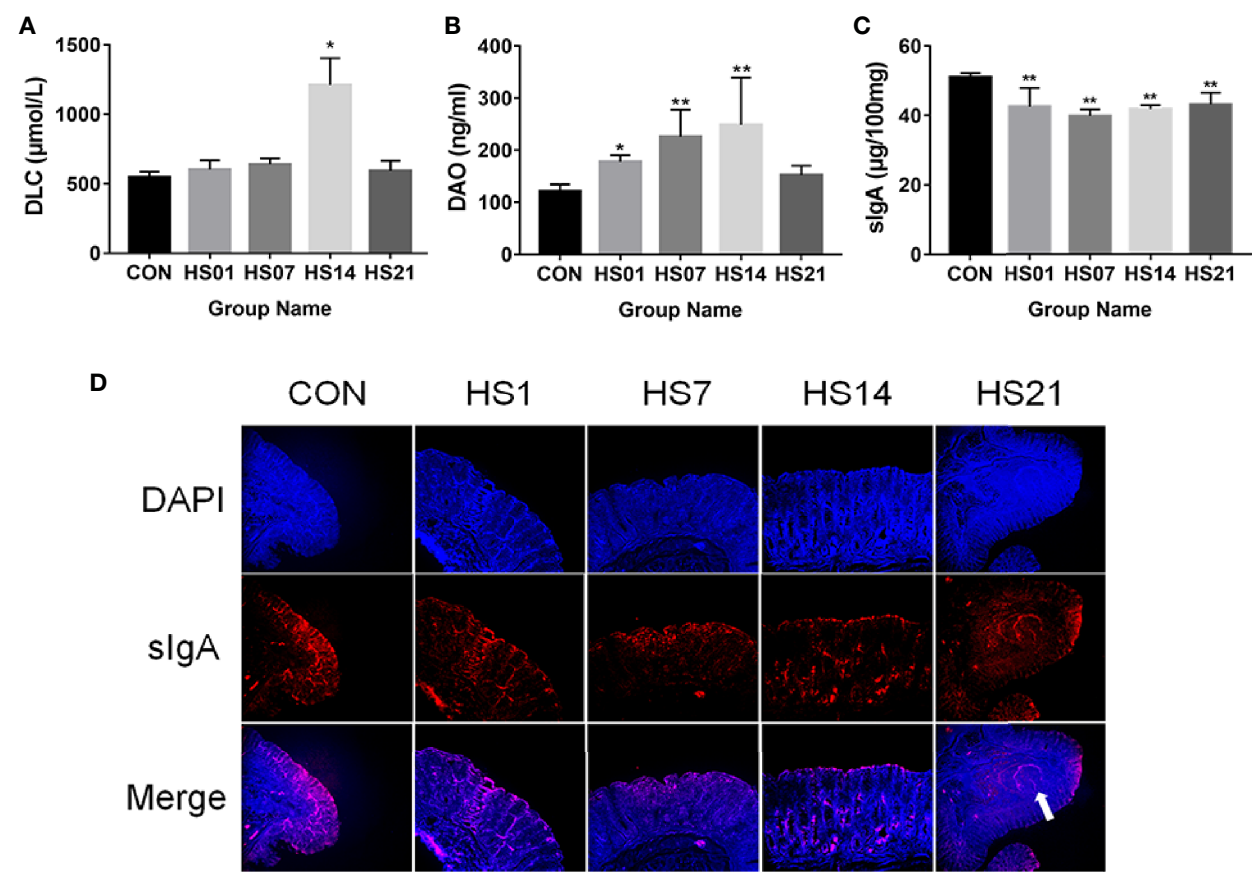

FIGURE 3 | Effect of HS on the biochemical markers of serum and colon. CON indicates no heat stress group of day 1, HS1, 7, 14, 21 indicate heat stress day 1, 7, 14, and 21 groups respectively, $n=6$; "*” indicates $p<0.05$, and "**” indicates $p<0.01$. (A) serum D-lactic acid; (B) serum diamine oxidase; (C) slgA of colonic content; (D) distribution of slgA in colon. 


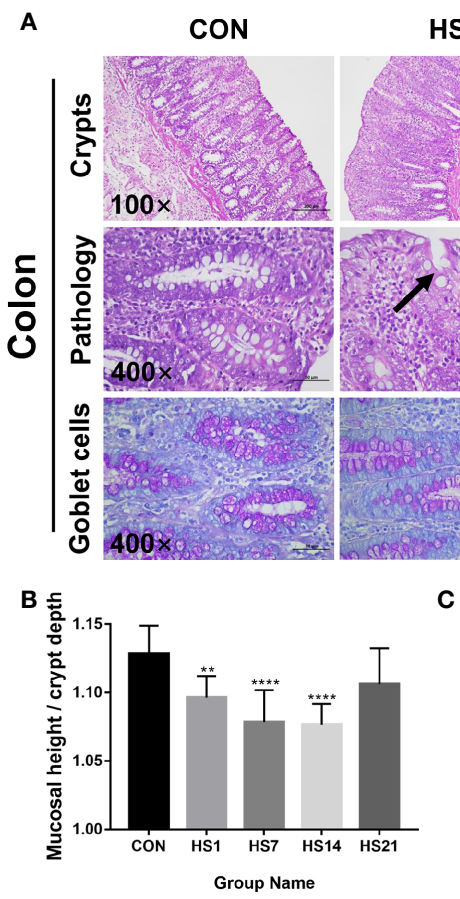

HS1

HS7

HS14

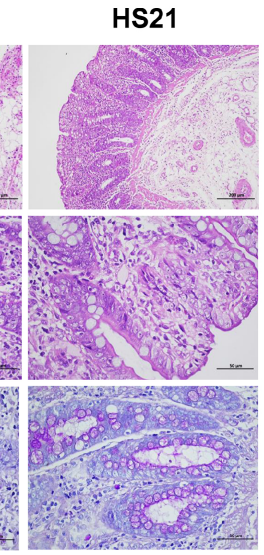

C

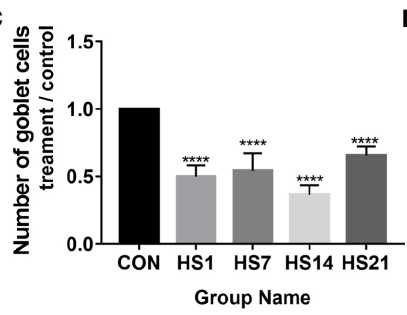

D

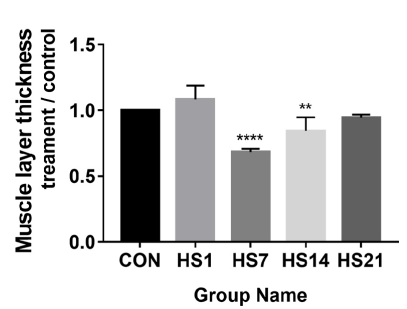

FIGURE 4 | Effect of HS on the morphology structure of colon. CON indicates no heat stress group, HS1, 7, 14, 21 indicate heat stress day 1, 7, 14, and 21

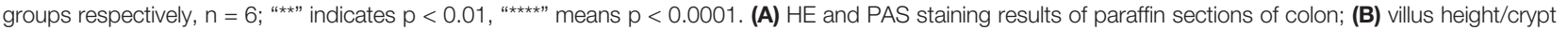
depth; (C) PAS positive cell number; (D) intestinal muscle layer thickness. As the arrows showing, after HS treatment, the colonic mucosal were loosely arranged and showed signs of bleeding on days 1, 7 and 14 of HS, the local injury and lymphatic infiltration also had been found on days 7 and 14 of HS.

all samples had no abnormal outliers, among which the samples on days 1 and 14 of HS were far away from the control group (Figure 5H). The results of the orthogonal projections to latent structures discriminant analysis (OPLS-DA) can effectively reduce the complexity of the model and enhance the explanatory ability of the model without reducing the predictive ability of the model, so as to maximize the difference between groups. OPLS-DA showed that there was significant difference between the HS group and the control group, except on day 7 of HS (Figure 5I). The results of metabolic difference analysis between SCFAs showed that there was a strong positive correlation (correlation coefficient more tended to 1) between acetic acid, propionic acid, butyric acid and valeric acid, and a positive correlation was observed between isobutyric acid and isovaleric acid (Figure 5J). Z-score (standard score) was calculated based on the content of metabolites, which is used to measure the content of metabolites at the same level. The results of Z-score analysis showed that the metabolite content in the HS group on days 1 and 14 was significantly lower than that in the control group, and the content of SCFA metabolites in the colons of pigs on day 21 was slightly higher than that in the control group (Figure 5K).

\section{Effect of HS on the Microbiome of Pig Colon}

The results of alpha diversity index showed that the homogeneity of samples in each group was high, and the Shannon index in day
7 of HS group was significantly $(P<0.05)$ lower than that in control groups, while Simpson index in day 14 of HS group was significantly $(P<0.01)$ lower than that in day 14 of control groups. The Chao and Ace indexes in day 7, 14 and 21 of HS group was significantly $(P<0.01)$ higher than that in each control groups (Figure 6A). PCA analysis showed that there was a significant difference in the flora of the HS and control groups (Figure 6B). The cluster thermogram (Figure 6C) showed that the distance between HS groups in different time was closer, and the cluster relationship between the control groups in different time was closer.

At the phylum level, all groups contained ten phylum level microorganisms, namely Firmicutes, Bacteroidetes, Spirochaetes, Actinobacteria, Proteobacteria, Chlamydiae, Patescibacteria, Tenericutes, Cyanobacteria, and Euryarchaeota; Among them, Firmicutes and Bacteroides were predominant, and their total number accounts for more than 60\% (Figure 7A). Venn analysis showed that there was among the same 121 genera in each group (Figure 7D). The top 5 bacteria by genus level in the total distribution were Lactobacillus (the largest proportion is greater than 30\%), Prevotella $(2-20 \%)$, Clostridium (the largest proportion is greater than 27\%), Streptococcus (2-20\%), and Treponema $(0-11 \%$, Figure $7 \mathbf{B})$. The bacteria in the first 5 bacteria genus levels had a relatively high abundance of $5 \%$ were observed as shown (Figure 7F). The percentage of Lactobacillus decreased significantly (decreased by more than $16 \%$ and $14 \%$ by 

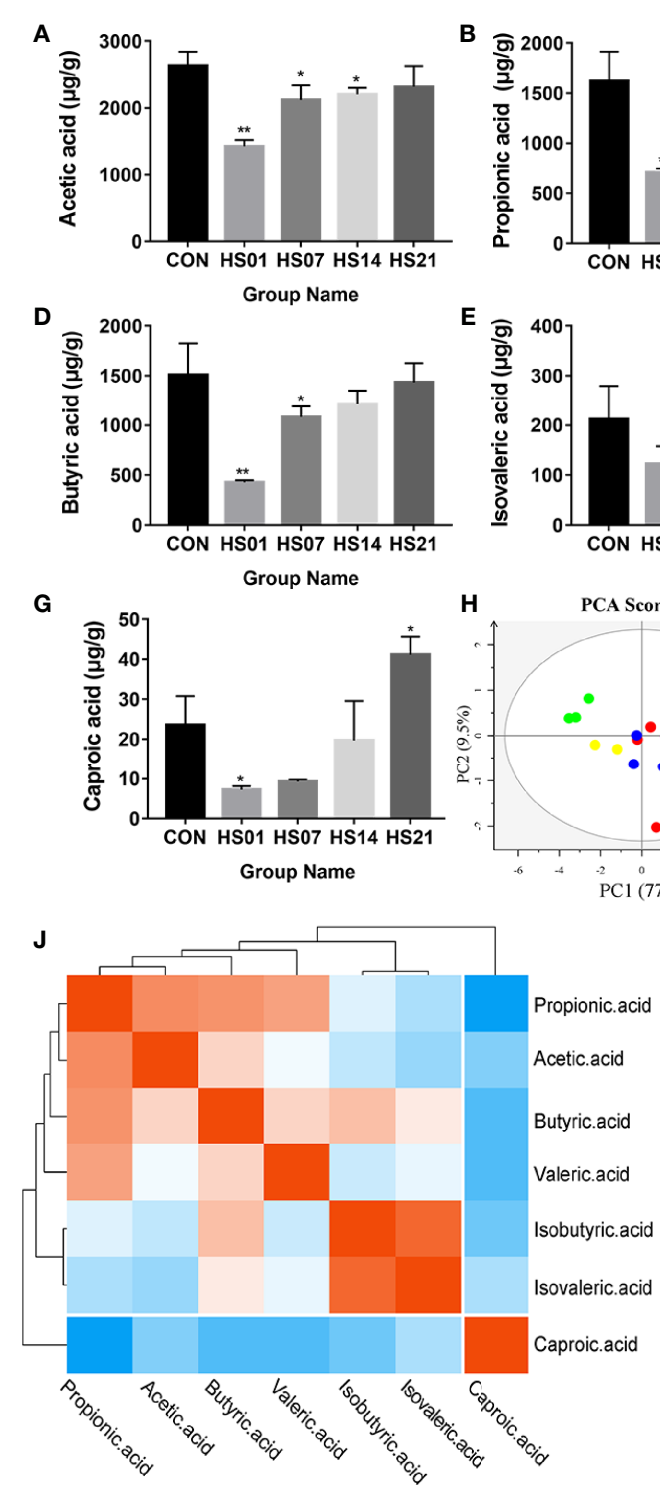
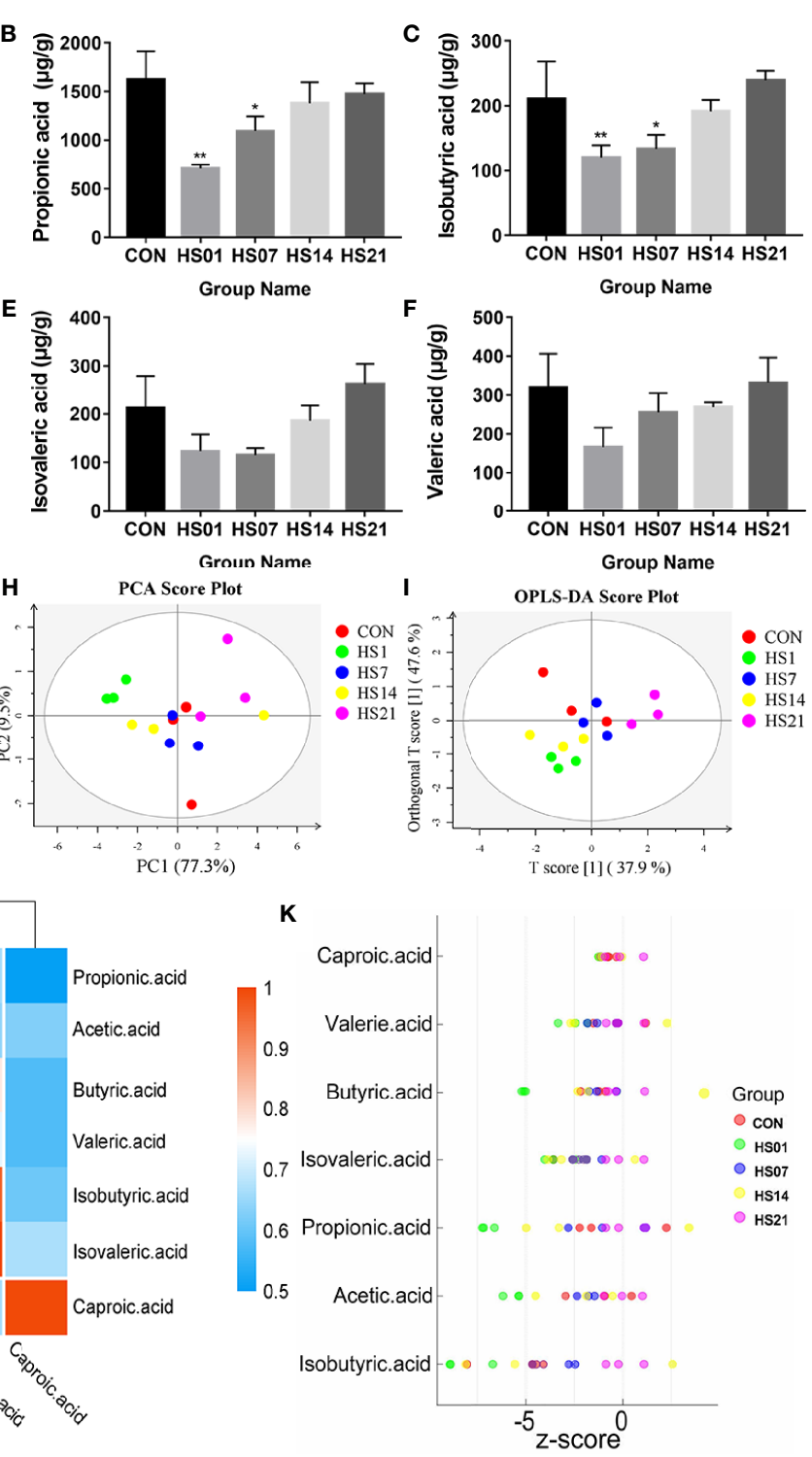

FIGURE 5 | Effect of HS on the metabolism of SCFAs in pig colon. CON indicates no heat stress group of day 1, HS1, 7, 14, 21 indicate heat stress day 1, 7, 14, and 21 groups respectively, $n=4$; “*” indicates $p<0.05$, and "**” indicates $p<0.01$. (A) acetic acid; (B) propionic acid; (C) isobutyric acid; (D) butyric acid;

(E) isovaleric acid; (F) valeric acid; (G) caproic acid; (H) PCA analysis between short-chain fatty acid samples; (I) short-chain OPLS-DA analysis between fatty acid samples; (J) metabolite correlation analysis; (K) Z-score analysis.

compared with each control group) on the days 14 and 21 of HS, as well as the Prevotella by compared with each control group (decreased by $12 \%, 9 \%$ and $3 \%$ respectively on day 7,14 and 21 , $\mathrm{P}<0.05)$; whereas the percentage of Clostridium increased by more than $11 \%$ after HS treatment on day 14 and 21 . Kineothrix decreased significantly (more than $4 \%, \mathrm{P}<0.05$ ) on day 1 of $\mathrm{HS}$, and Oscillibacter also decreased significantly (more than $2 \%, \mathrm{P}<$ 0.05) on days 1,14 and 21 of HS.

Venn analysis also showed that there was among the same 157 species in each group, and day 1 of HS possessed 15 unique species (Figure 7E). The top 5 bacteria by species level in the total distribution were Lactobacillus johnsonii, uncultured bacterium g Clostridium, Lactobacillus amylovorus, Streptococcus gallolyticus, and Lactobacillus reuteri (Figure 7C). The bacteria in the first 5 bacteria species levels had a relatively high abundance of 5\% were observed as shown (Figure 7G). The percentage of Lactobacillus johnsonii was decreased significantly $(P<0.01)$ on the days 7 and 14 of HS, $12.4 \%$ and $18.3 \%$ respectively, by compared with each control group, Lactobacillus amylovorus was increased $8.63 \%$ in day 7 of HS; whereas the percentage of uncultured bacterium $g$ Clostridium increased after HS treatment (increases by $15.5 \%, 4.0 \%, 10.1 \%$, and $9.2 \%$ respectively, $P<0.05)$. Kineothrix alysoides decreased significantly $(4.00 \%, P<0.05)$ on day 1 of HS, and Lactobacillus 
A

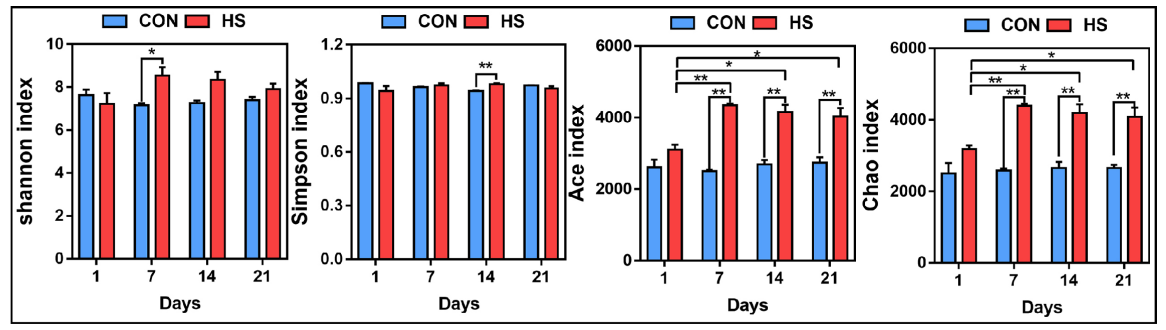

B

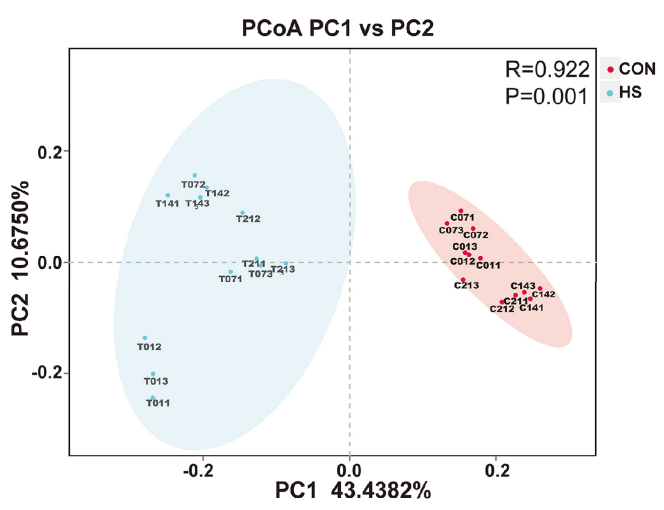

C

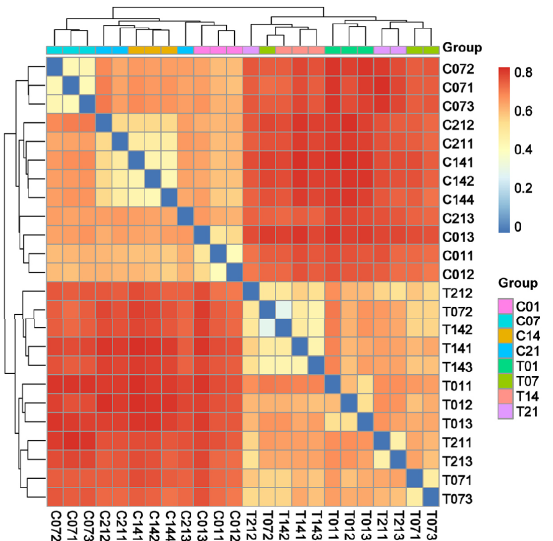

FIGURE 6 | The effect of heat stress on the microbial diversity of pig colon. CON and C indicate no heat stress group, HS and T indicate heat stress treatment, 1, 7, 14, 21 indicate day 1, 7, 14, and 21 groups respectively, $n=3$; "** indicates $p<0.05$, and "**" indicates $p<0.01$. (A) inter-group Alpha diversity analysis of Shannon index, Simpson index, Ace index and Chao Index; (B) principal component analysis between samples; (C) species composition heat map between samples.

reuteri also decreased significantly $(4.5 \%, P<0.05)$ on day 21 of HS.

We compared the differences of dominant bacteria (genus and species) between HS group and control group on the days 1, 7 and 14 (Figure 8A). The dominant bacteria in the control group of day 1 were Lactobacillales johnsonii, Rikenellaceae RC9 gut group, Kineothrix alysoides and Prevotellaceae, etc; in the control group of day 7, dominant bacteria were Lactobacillus reuteri, Prevotella copri, Rikenellaceae RC9 gut group, Lactobacillales johnsonii and Treponema 2, etc.; While the dominant bacteria in the control group of day 14, also had Prevotellaceae, Lactobacillales johnsonii and Rikenellaceae RC9 gut group. In contrast, the dominant bacteria on days 1,7 and 14 of HS group were Clostridium sensu stricto 1 , while on day 14, the dominant bacteria of HS group also had Terrisporobacter and Turicibacter sanguinis, etc., indicating that HS could significantly change the microbial structure and increase the content of opportunistic pathogens (Figures 8B, C). The KEGG difference prediction analysis based on PICRUST found that the colonic microbiota may have an impact on Environmental information processing and Cellular processes in pigs on the day 7 of HS by compared with its' control group $(P<0.01$, Figure 8D). And it also shows that colonic microbiota may affect pigs' genetic information processing in day 14 of HS $(P<0.01$, Figure 8E).

Redundancy analysis/canonical correspondence analysis (RDA/ CCA) were used to analyze the correlation among microbiome and SCFAs metabolites in each group. As shown in Figure 9A, the cumulative contribution rate of SCFAs to the structure of the flora was $9.78 \%$ and $15.39 \%$, respectively. We found that the abundance of Turicibacter sangunis was negatively correlated with SCFAs metabolism, whereas the changes in the abundance of Megasphaera of was positively correlated with SCFAs metabolism. In addition, the abundance of Terrisporobacter mayombei, Lactobacillus reuteri, lactobacterium succinatutens were negatively correlated with isobutyric acid, valeric and caproic, but positive correlated with butyric. There was a positive correlation between the metabolism of isobutyric acid, valeric and caproic and that of Kineothrix alysoides, Treponema procinum and Lactobacillus amylovorus. In addition, there was a positive correlation between Megasphaera of and SCFAs metabolism, indicating the beneficial role of Megasphaera $c f$ in fatty acid metabolism. In contrast, there was a negative correlation between Ruminococcaceae and SCFAs, except propionic acid, indicating its negative regulatory role in SCFAs metabolism. In addition, in terms of flora composition and SCFAs metabolism composition, there was a pairwise comparison of the negative correlation between isobutyric acid, isovaleric acid and Megasphaera cf, Turicibacter sanguinis, Terrisporobacter mayombei, and Ruminococcaceae UCG-005 (Figures 9A, D). As shown in Figures 9C-E, RDA showed that the cumulative contribution rate of serum biochemical index to the structure of the flora was $13.53 \%$ and $22.36 \%, 9.8 \%$ and $15.37 \%$, respectively. The changes in the percentage of Lactobacillus reuteri, Megasphaera cf, Phascolarctobacterium succinatutens and Lactobacillus johnsonii were positively correlated with the changes in serum total bilirubin 

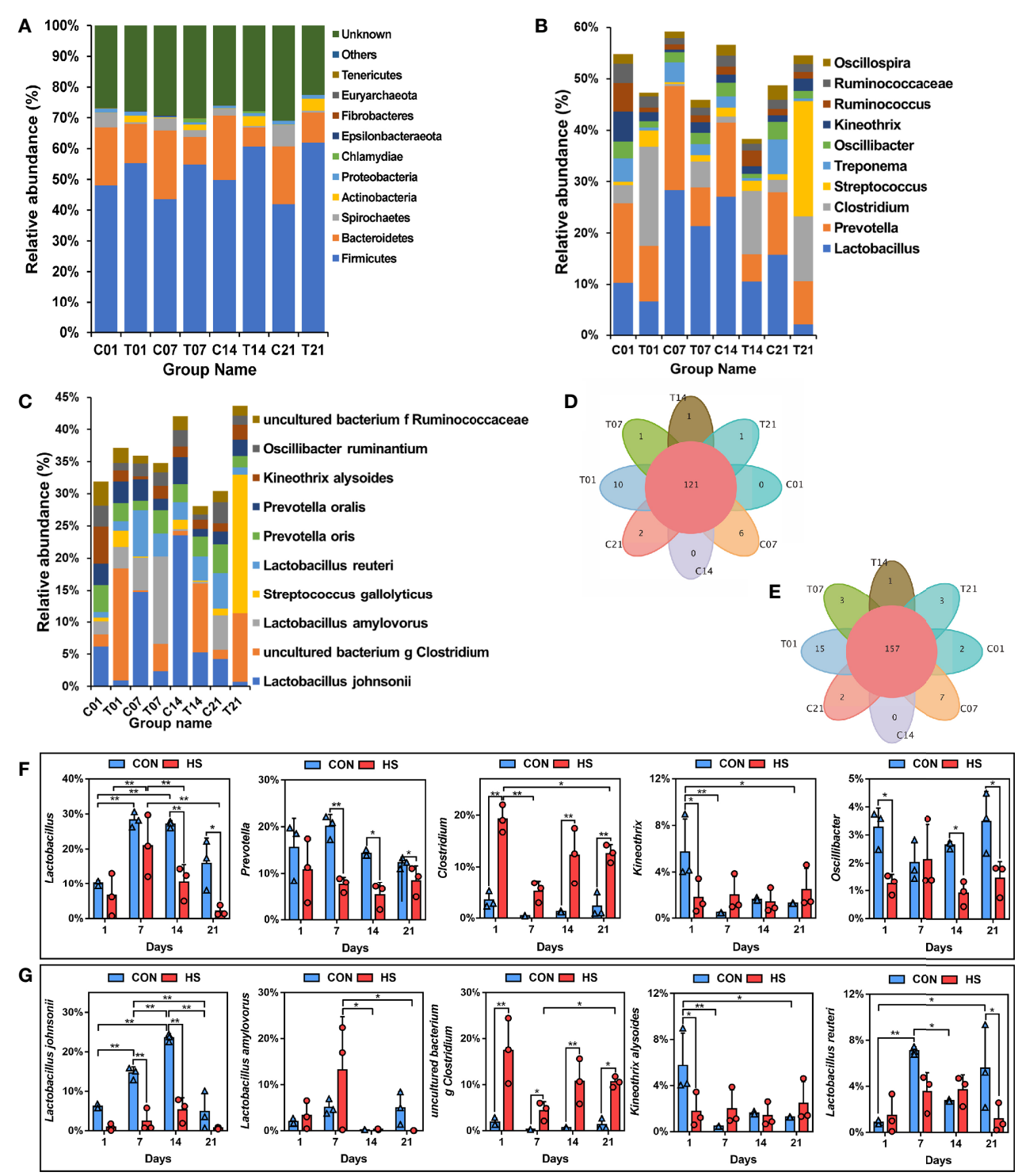

FIGURE 7 | Effect of HS on the microbial structure of pig colon. CON and C indicate no heat stress group, HS and T indicate heat stress treatment, 1, 7, 14, 21

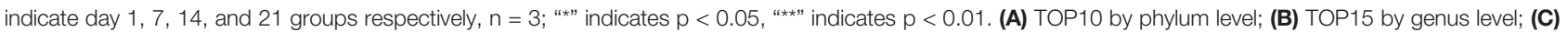
TOP15 by species level; (D) Venn plot of genus level; (E) Venn plot of species level; (F) differences of phylum level by TOP5; (G) differences of genus level by TOP5.

and the ratio of albumin and globulin, while were negatively correlated with the changes in serum creatinine, alanine transaminase, potassium ion, alkaline phosphatase and albumin. The changes in the percentage of Lactobacillus amylovorus, Kineothrix alysoides, Turicibacter sanguins and Treponema porcinum were positively correlated with the changes in serum chloride, alkaline phosphatase, potassium ion, alanine transaminase and creatinine, while were negatively correlated with the changes in serum globulin, total carbon dioxide, total bilirubin and the ratio of albumin and globulin (Figure 9B). Besides, abundance of Lactobacillus reuteri, Megasphaera cf, and Lactobacillus amylovorus have negative correlation with calcium ions, lactic acid, creatine kinase, amylase, inorganic phosphorus and total bilirubin; the abundance of Lactobacillus johnsonii, Treponema porcinum, Phascolarctobacterium succinatutens and Terrisporobacter mayorrbei have negative correlation with serum $\mathrm{pH}$, total protein, total cholesterol; while negatively correlated with glucose, lactate and creatine kinase. The concentrations of serum glucose was positively correlated with the Kineothrix alysoides. The abundance of Turricibacter sanguinis was positively correlated with lactic acid, creatine kinase, amylase, inorganic phosphorus (Figure 9C). Correlation heat map analysis found that Lactobacillus amyovorus has a negative correlation with liver and kidney metabolism in pigs, and Ruminococcaoeae UCG-014 have a negaitive correlation with liver and kidney metabolism in contrast. While Clostridium sensu stricto 1 has a positive correlation with 

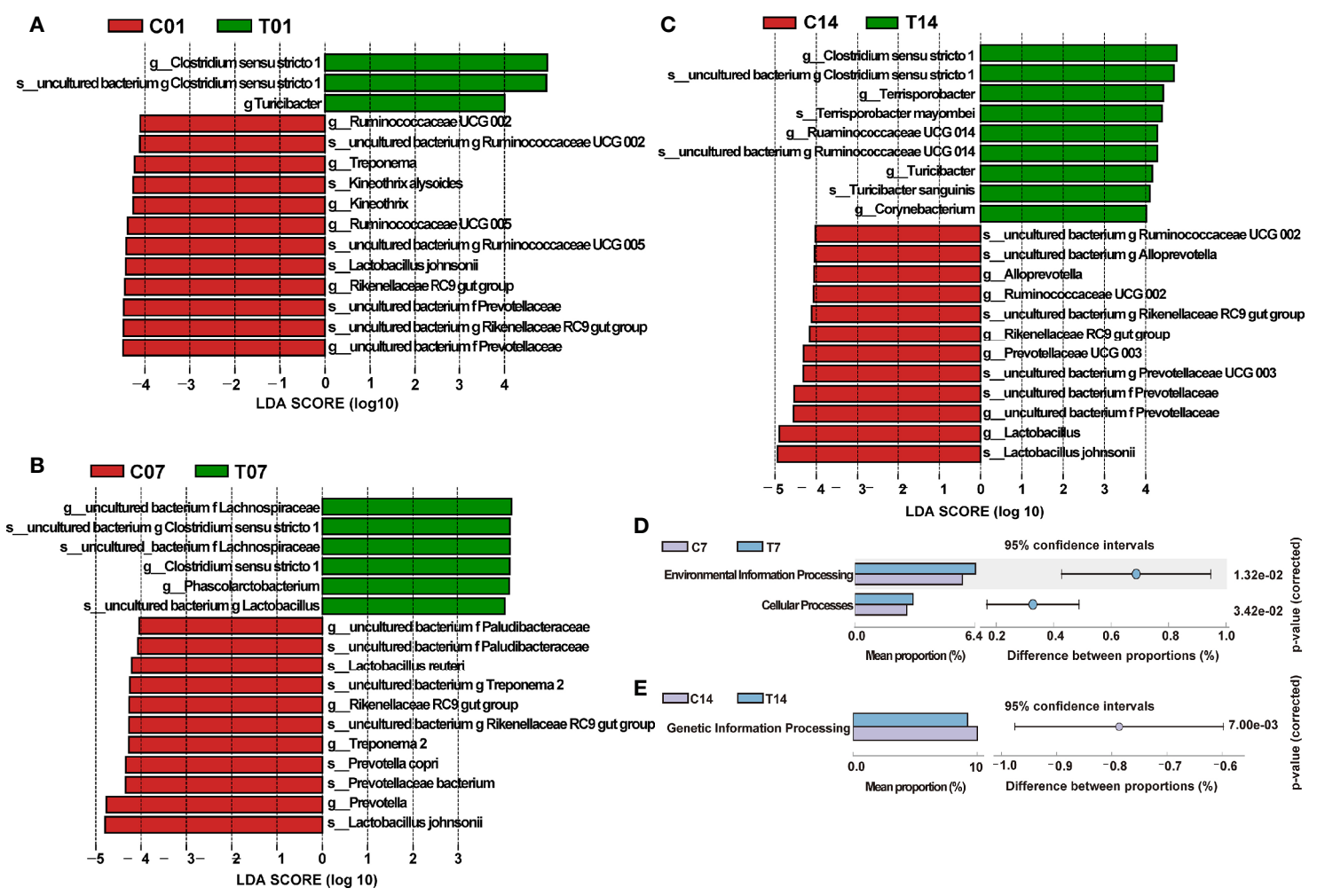

D

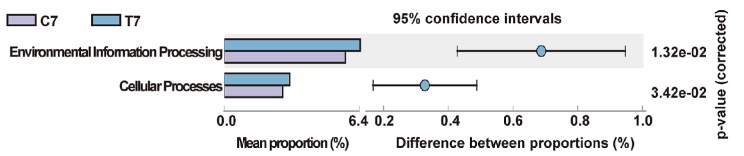

E $\square \mathrm{C} 14 \quad \square \mathrm{T} 14$

FIGURE 8 | Effect of HS on the difference in microbial composition of pig colon. CON and C indicate no heat stress group, HS and T indicate heat stress treatment, 1, 7, 14, 21 indicate day 1, 7, 14, and 21 groups respectively; (A) LEfSe analysis between C01 and T01 groups; (B) LEfSe analysis between C07 and T07 groups; (C) LEfSe analysis between C14 and T14 groups; (D, E) KEGG metabolism prediction differences between groups in day 7 and 14 of HS.

alanine aminotransferase, alkaline phosphatase, creatinine, amylase, inorganic phosphorus, lactic acid, creatine kinase and total cholesterol (Figures 9E, F).

As shown in Table 1, Mantel-test showed that acetic acid is the key $(\mathrm{R}=0.200197, P=0.043)$ to determine the microbial composition, whereas isobutyric acid and isovaleric acid were negatively correlated with the microbial composition. With respect to the biochemical indexes, the concentrations of lactic acid, amylase, creatine kinase, and potassium ion, and $\mathrm{pH}$ of the serum were positively correlated with the changes in colonic flora structure $(P<0.05)$.

\section{Transcription Sequencing Analysis of Colon Tissue in HS Pigs}

From the sequencing results, we found that the correlation heat map between the samples showed that the distances of samples in HS groups were close (Figure 10A). Based on the differential expression volcanic plots, we found that when the difference threshold (HS/control) was 2, on days 1, 7, 14, and 21, 92, 387, 555 , and 461 genes were upregulated $(P<0.05)$, respectively, while 60, 483, 549, and 467 genes were down-regulated, respectively (Figures 10B-E).

Venn analysis of the genes in the control group and HS groups on days 7 and 14 showed that there were 555 genes that significantly changed in HS pigs (Figure 10F). These genes were enriched in the immune-related signaling pathways, such as the nucleotide-binding oligomerization domain (NOD)-like receptor signaling pathway, systemic lupus erythematosus, cytokine and cytokine receptor interaction, Th17 cell differentiation signaling pathway, janus kinase (JAK)- signal transducer and activator of transcription (STAT) signaling pathway, Th1 and Th2 cell differentiation, the forkhead box $\mathrm{O}$ signaling pathway, and IBD signaling pathway. In addition, related functions such as Alcoholism Viral carcinogenesis, Hepatitis B, Mismatch repair and Amoebiasis pathways were also enriched by analysis (Figure 10G).

After a co-expression weighted association analysis for the 555 genes (transcripts) and SCFAs, we found that 327 genes (transcripts) were associated with SCFAs metabolism. Based on the modules in blue, we constructed an interaction network of pathways using data from GO and KEGG database, and the 152 genes were annotated. Specifically, the gray module was utilized for housing the genes that were not co-expressed with other genes and thus could not be assigned to any of the other modules; we have ignored these in in our study. In addition, according to the green module, a total of 41 known genes were significantly related to the metabolites of SCFAs. Among the green module genes, glucagon like peptide receptor (GLP1R) and collagen lectin family protein 11 were significantly associated with SCFAs metabolism (Figures 10H-I). 


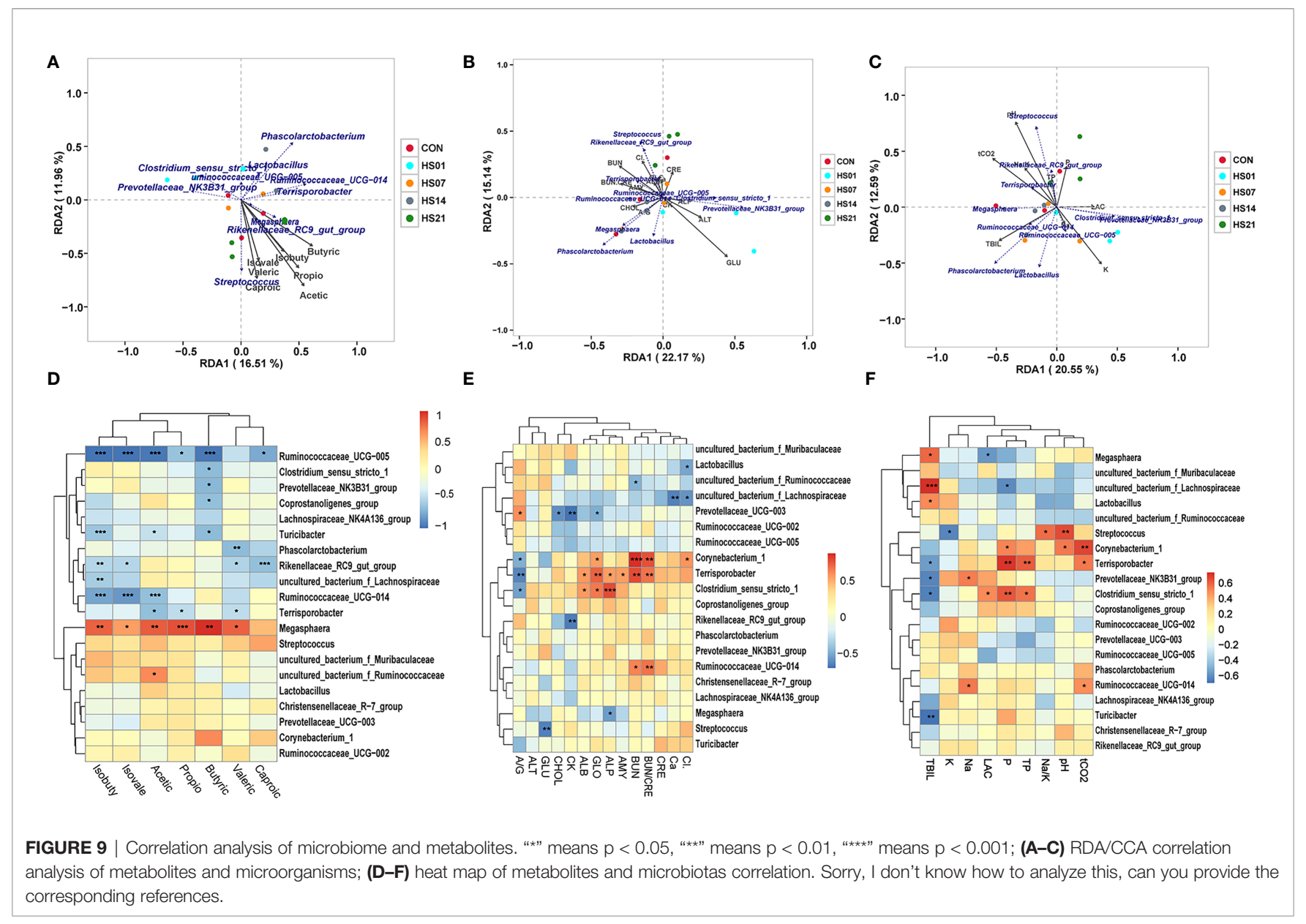

TABLE 1 | Correlation analysis of microorganisms and metabolites Mantel-test.

\begin{tabular}{|c|c|c|c|c|c|}
\hline Environmental factors & Mantel-test R statistic & $\mathrm{p}$-Value & Environmental factors & Mantel-test R statistic & $\mathrm{p}$-Value \\
\hline Propionic & 0.146603 & 0.102 & $\mathrm{Na}^{+}$ & -0.00882 & 0.504 \\
\hline Butyric & 0.149879 & 0.108 & $\mathrm{Na}^{+} / \mathrm{K}^{+}$ & 0.172682 & 0.077 \\
\hline Isovaleric & -0.10435 & 0.822 & BUN & -0.07445 & 0.754 \\
\hline Valeric & 0.086125 & 0.213 & CRE & -0.02208 & 0.564 \\
\hline Caproic & 0.109714 & 0.209 & BUN/CRE & -0.17751 & 0.938 \\
\hline $\mathrm{CHOL}$ & 0.121927 & 0.168 & $\mathrm{CK}$ & 0.225902 & 0.032 \\
\hline LAC & 0.213002 & 0.048 & ALP & 0.159538 & 0.073 \\
\hline $\mathrm{pH}$ & 0.245673 & 0.021 & ALT & 0.120687 & 0.189 \\
\hline $\mathrm{P}$ & 0.07994 & 0.2 & ALB & 0.022509 & 0.409 \\
\hline $\mathrm{Cl}^{-}$ & 0.138532 & 0.123 & GLO & -0.05131 & 0.687 \\
\hline TP & -0.01936 & 0.545 & $A / G$ & -0.05389 & 0.665 \\
\hline
\end{tabular}

Using the WGCNA analysis for the correlation between serum biochemical indexes and the above 555 genes (transcripts), we first eliminated the outlier indexes, total bilirubin and $\mathrm{A} / \mathrm{G}$, as they had no correlation with the transcripts (Figure 11A). Next, the significant changed genes (transcripts) in the HS group on days 7 and 14 were divided into four modules, according to their correlation trend (Figures 11B, C). Based on these three modules-green, tan, and blue, (the gray module was utilized for 

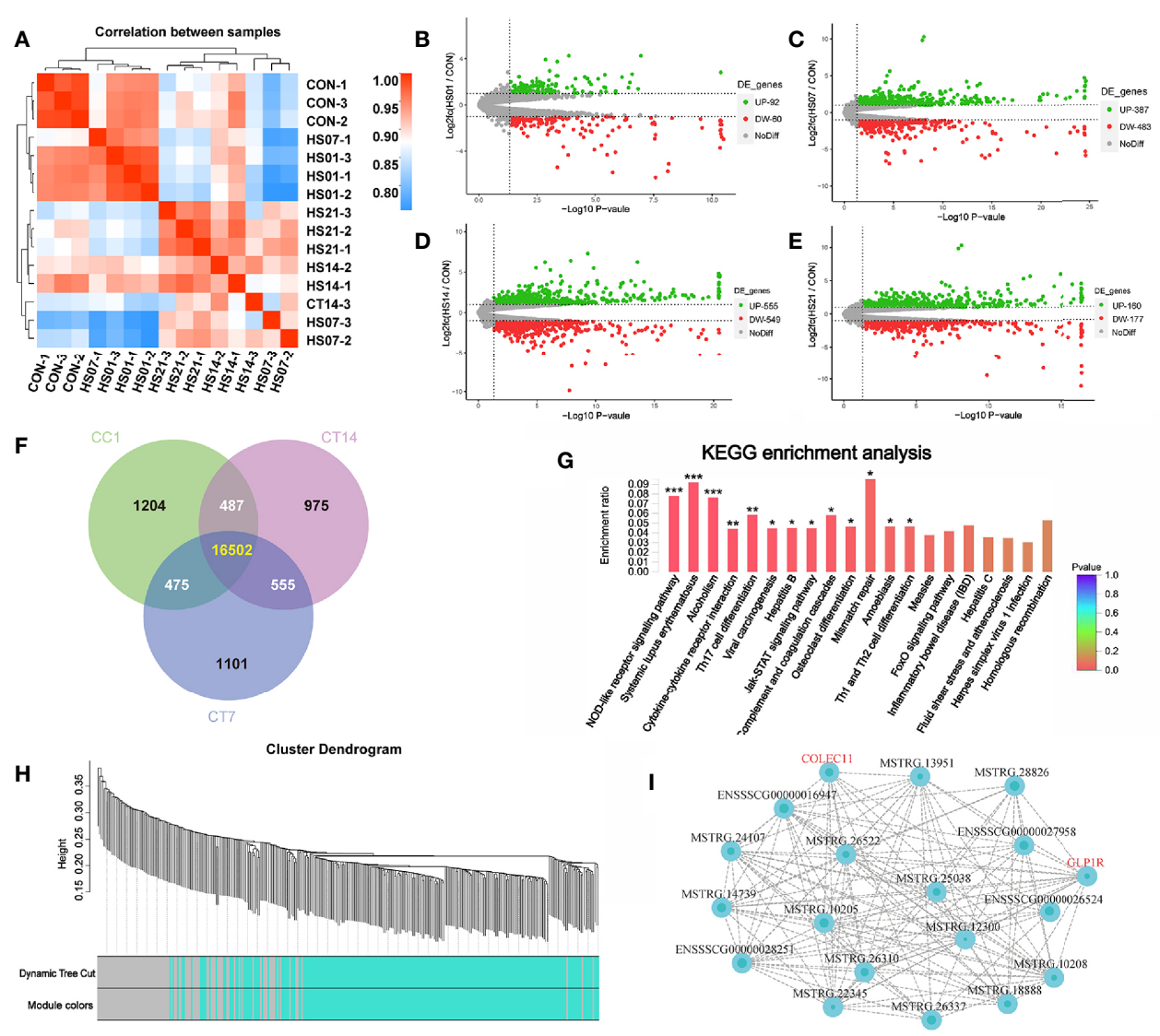

G
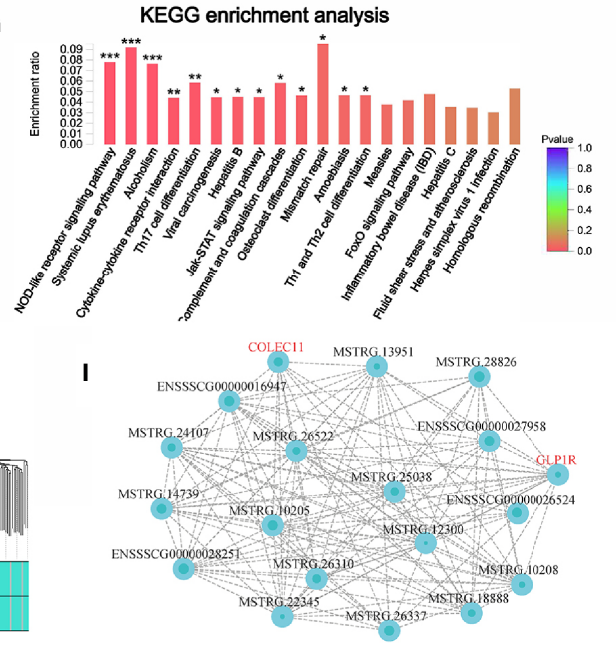

FIGURE 10 | Transcription sequencing analysis of HS pig colon. CON indicates no heat stress group of day 1, HS1, 7, 14, 21 indicate heat stress day 1, 7, 14, and 21 groups,

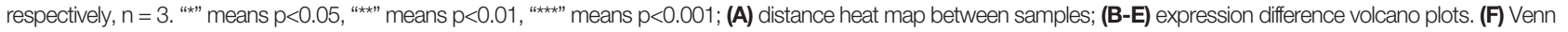
analysis between groups, (G) difference gene KEGG enrichment analysis; (H) WGCNA modular analysis; (I) WGCNA module related gene interaction network of green module.

housing the genes that were not co-expressed with other genes, we have ignored these in study.) we constructed an interaction network of pathways using data from GO and KEGG database. The blue module could recognize the interaction network of neuron-specific vesicular protein calcyon, testis-specific serine kinase, cellular retinoic acid-binding protein 1, sialoadhesin, and serum biochemical indicators (Figure 11D). The green module could recognize matrix metalloproteinase-12, cell adhesion molecule 2, adhesion $G$ protein coupled receptor E1, triggering receptor expressed on myeloid cells 2, transcription factor 6, potassium voltage-gated channel delayed-rectifier subfamily $\mathrm{S}$ member 1 , hydrogen potassium exchange ATP and subunit $\beta$, and the interaction network of $A T P 4 B$ and other genes with serum biochemical indicators (Figure 11E).

\section{DISCUSSION}

When animals are exposed to high temperature environment, water loss by evaporation and heat dissipation of the body through the epidermis is increased, which may lead to electrolyte imbalance (38-40). This in turn may decrease the blood sodium and chloride ion concentration $(41,42)$, and thereby affect the functioning of the urinary system. Furthermore, water loss may lead to dehydration, affecting the normal function of the kidney (43). In this study, the total protein, albumin, and the concentration of serum creatinine were increased in HS pigs, indicating that electrolyte balance and renal function were affected by HS. Furthermore, some biochemical indexes related to liver function, such as globulin, alanine aminotransferase, and cholesterol, also increased in HS pigs, suggesting that the liver metabolic function changes under HS. The interaction between calcium and chloride ions has been reported to affect the intestinal barrier function and transportation of nutrients $(44,45)$.

Alkaline phosphatase not only plays an important role in the absorption of calcium, fatty acids, and other minerals or nutrients, but also can prevents the invade of bacterial components Lipopolysaccharide (LPS) and flagellin (46-48). When alkaline phosphatase binds to intestinal epithelial cells, it inhibits the growth of potential pathogens, such as Escherichia coli (49). In addition, alkaline phosphatase and the secreted heat shock proteins participate in the colonization of intestinal microorganisms in piglets and play an important role in the production of antioxidant enzymes and anti-inflammatory and 

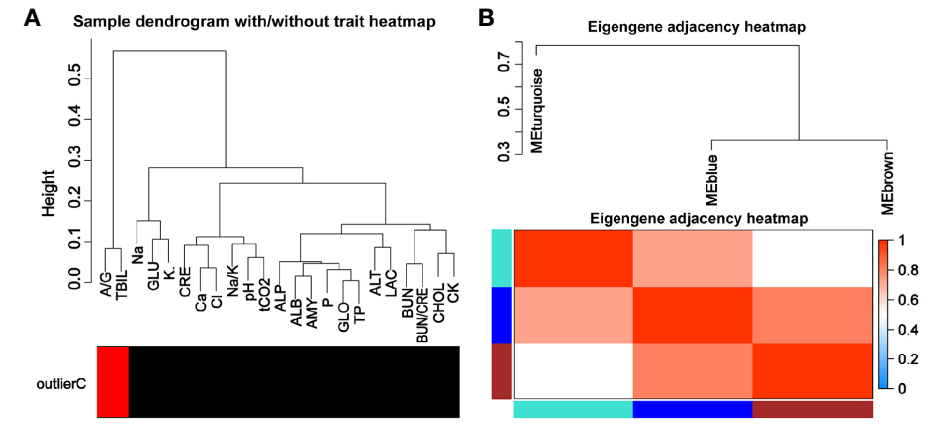

C

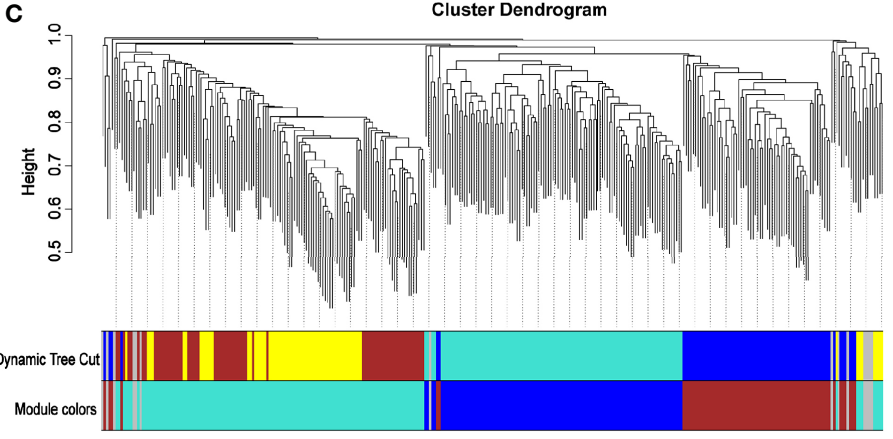

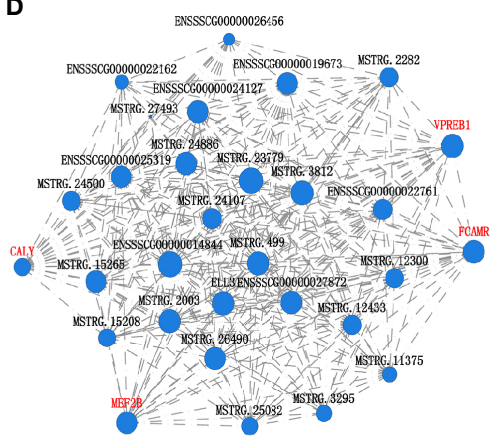

E

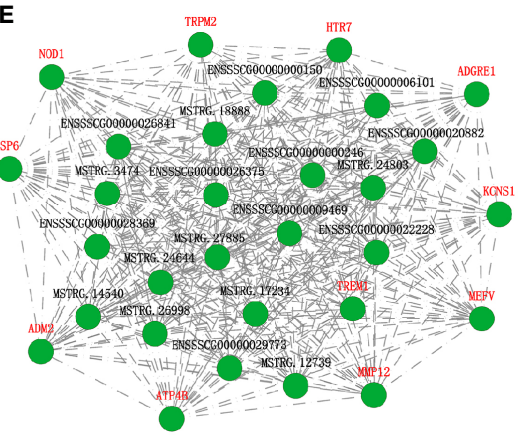

FIGURE 11 | Correlation analysis of heat-stressed pig colonic transcriptome and serum biochemical indicators. (A) WGCNA cluster analysis; (B, C) WGCNA modular analysis; (D, E) WGCNA module related gene interaction network.

regulation of the intestinal function $(46,50,51)$. In this study, alkaline phosphatase increased significantly after HS treatment. We hypothesized that the damage to intestinal balance induced the regulatory function of host and resulted in the upregulation of alkaline phosphatase.

Diamine oxidase and D-lactic acid are used as clinical indicators of enteritis, intestinal tissue, and barrier damage $(52,53)$. We found that both diamine oxidase and D-lactic acid increased in HS pigs, indicating that the occurrence of intestinal damage. Analysis of the morphological structure showed that villus height/crypt depth decreased significantly in the gut of HS pigs, and the thickness of intestinal muscle layer decreased. Under normal physiological conditions, the mucous layer covers the microvilli of epithelial cells, which is the first protective barrier to prevent intestinal microorganisms from entering the mucosal layer (54-56). Goblet cells are the main source of mucus, and a decrease in goblet cells has been reported to result in the invasion of bacteria (56). sIgA is an important physical barrier in the intestine. It can inhibit the adhesion and colonization or invasion of pathogenic microorganisms to the surface of intestinal mucosa (20). In HS pigs, sIgA was significantly reduced in both colon tissues and contents, suggesting a serious intestinal barrier injury.

Intestinal microflora participate in the digestion of carbohydrates and produce SCFAs, which protect the epithelium from injury, participate in the synthesis of essential amino acids, regulate lipid metabolism, induce intestinal peristalsis, improve and regulate intestinal angiogenesis, and activate of the immune system $(57,58)$.
In addition, SCFAs regulate the production, transportation, and function of innate and adaptive immune cells. Butyric acid is a type of SFA that can directly act on the immune cells in the intestinal mucosa, increase the number and activity of Treg, and inhibit the activity of neutrophils, macrophages, dendritic cells, and effector T cells (59). It is commonly used as a marker of intestinal homeostasis (60). We found that under HS, the content of butyric acid in colonic content decreased significantly, in addition to the decrease in acetic acid, propionic acid, and hexanoic acid, thus indicating that HS affected the metabolism of SCFAs in pigs.

The dominant bacteria in the control group were beneficial bacteria, such as Lactobacillus. while the dominant bacteria in HS group were pathogenic bacteria, such as Clostridium sensu stricto 1 and Terrisporobacter, which play a vital role in the occurrence of intestinal inflammation (61-63). Correlation analysis with serum biochemical indexes showed that Phascolarctobacterium, Lactobacillus and Megasphaera $c f$ were positively correlated with the concentrations of serum cholesterol, creatine kinase, and total bilirubin, while negatively correlated with the concentrations of lactic acid, creatinine, sodium ion, albumin, globulin, and alkaline phosphatase, thus suggesting that intestinal beneficial bacteria can maintain the health of the pigs under normal condition. Clostridium sensu stricto 1 is an opportunistic pathogen $(63,64)$, which can cause intestinal inflammation and decrease the content of SCFAs (65). In our study, we found that this pathogen was significantly increased on day 1 of HS, and was negatively correlated with serum total 
bilirubin, and positively correlated with the content of lactic acid, inorganic phosphorus, total protein, globulin, albumin, and alkaline phosphatase, thus indicating that it may be involved in the occurrence of intestinal inflammation in HS-pigs.

The level of SCFAs and microbial structure were correlated closely (66). In this study, the cumulative contribution rate of SCFAs to the structure of the flora was $9.78 \%$ and $15.39 \%$, respectively. We found that the abundance of Turicibacter sangunis was negatively correlated with SCFAs metabolism. Although Megasphaera $c f$ was positively correlated with SCFAs metabolism, Clostridium sensu stricto 1 was negatively correlated with SCFAs except propionic acid. We hypothesized that the change in dominant bacteria could change the metabolism level of SCFAs. Gonçalves et al. found that there is a positive regulatory relationship between the metabolism of Phascolarctobacterium and Lactobacillus and the metabolism of acetic acid and butyrate (59), which is consistent with the findings of our study.

HS can significantly change the expression of multiple genes (transcripts), which are enriched in many inflammatory immune related signaling pathways, such as toll like receptor signal, interleukin (IL)-17 signal, NOD-like receptor signal, and JAKSTAT signal. In metabolite transcriptome association analysis, glucagon like peptide receptor (GLP1R), which is related to SCFAs metabolism, can activate nuclear factor kappa light chain enhancer of activated B cells signaling pathway (67) and Myosin light-chain (68) via cAMP, leading to inflammation and intestinal barrier damage, indicating that the metabolism of SCFAs is related to HS-induced intestinal inflammation. This finding can be considered as a breakthrough point for further studies on the pathogenesis of IBD in pig.

In this study, we ignored the time effect in the setting of the control group (Supplementary Material 2), only designed the heat stress effect in bacteria diversity, SCFA, and transcriptome. We found that the structure, diversity, and metabolite levels of intestinal microorganisms in HS-pigs changed significantly, which led to the activation of immune response and inflammation signal pathways and caused abnormal physiological and biochemical indexes and intestinal mucosal damage in pigs. The results provide useful information to understand the important of intestinal flora in stress induced IBD and suggest that maintenance of intestinal flora balance may be a useful technique for IBD therapy.

\section{HEALTH AND SAFETY}

All our experimental procedures were conducted according to the universally accepted codes of laboratory practice and/or as

\section{REFERENCES}

1. Nagao-Kitamoto H, Shreiner AB, Gillilland MG, Kitamoto 3S, Ishii C, Hirayama A, et al. Functional Characterization of Inflammatory Bowel Disease-Associated Gut Dysbiosis in Gnotobiotic Mice. Cell Mol Gastroenterol Hepatol (2016) 2(4):468-81. doi: 10.1016/j.jcmgh.2016.02.003

2. Kiesler P, Fuss IJ, Strober W. Experimental Models of Inflammatory Bowel Diseases. Cell Mol Gastroenterol Hepatol (2015) 1(2):154-70. doi: 10.1016/j.jcmgh.2015.01.006 per manufacturer's instructions and followed all health and safety guidelines advocated by them.

\section{DATA AVAILABILITY STATEMENT}

The datasets used and/or analyzed during the current study are available from the corresponding author upon reasonable request.

\section{ETHICS STATEMENT}

The animal study was reviewed and approved by Animal Care and Use Committee of Guangdong Ocean University, Zhanjiang, China (Permit No. 206-1108).

\section{AUTHOR CONTRIBUTIONS}

Author order was determined both alphabetically and in order of increasing seniority. $\mathrm{XJ}$ conceived the project and designed the experiments. $\mathrm{CH}, \mathrm{XN}, \mathrm{SC}, \mathrm{JW}, \mathrm{MB}, \mathrm{SM}$, and LW conducted the experimental work and analyzed the data. XJ interpreted the results. $\mathrm{CH}$ prepared the figures and wrote the manuscript. XJ and YY edited the manuscript. XM, XL and ZY participated in the enrichment analysis, manuscript writing and revision. All authors contributed to the article and approved the submitted version.

\section{FUNDING}

This study was supported by the National Natural Science Foundation of China [grant numbers 31472243, 31902314]; Natural Science Foundation of Guangdong Province, China [grant number: 2019A1515011142]; the Project of Enhancing School with Innovation of Guangdong Ocean University [grant number: GDOU230419057]; the Basic Research Project of Shenzhen Science and Technology Innovation Commission (JCYJ20190813142005766).

\section{SUPPLEMENTARY MATERIAL}

The Supplementary Material for this article can be found online at: https://www.frontiersin.org/articles/10.3389/fimmu.2021.717723/ full\#supplementary-material

3. Kuda T, Yokota Y, Shikano A, Takei M, Takahashi H, Kimura B. Dietary and Lifestyle Disease Indices and Caecal Microbiota in High Fat Diet, Dietary Fibre Free Diet, or DSS Induced IBD Models in ICR Mice. J Funct Foods (2017) 35:605-14. doi: 10.1016/j.jff.2017.06.030

4. van Heugten E, Coffey MT, Spears JW. Effects of Immune Challenge, Dietary Energy Density, and Source of Energy on Performance and Immunity in Weanling Pigs. J Anim Sci (1996) 74(10):2431-40. doi: 10.2527/1996. 74102431x 
5. Pearce SC, Gabler NK, Ross JW, Escobar J, Patience JF, Rhoads RP, et al. The Effects of Heat Stress and Plane of Nutrition on Metabolism in Growing Pigs. J Anim Sci (2013) 91(5):2108-18. doi: 10.2527/jas.2012-5738

6. Fasano A, Shea-Donohue T. Mechanisms of Disease: The Role of Intestinal Barrier Function in the Pathogenesis of Gastrointestinal Autoimmune Diseases. Nat Clin Pract Gastroenterol Hepatol (2005) 2(9):416-22. doi: 10.1038/ncpgasthep0259

7. Krack A, Sharma R, Figulla HR, Anker SD. The Importance of the Gastrointestinal System in the Pathogenesis of Heart Failure. Eur Heart $J$ (2005) 26(22):2368-74. doi: 10.1093/eurheartj/ehi389

8. Pearce SC, Mani V, Boddicker RL, Johnson JS, Weber TE, Ross JW, et al. Heat Stress Reduces Barrier Function and Alters Intestinal Metabolism in Growing Pigs. J Anim Sci (2012) 90 Suppl:4, 257-9. doi: 10.2527/jas.52339

9. Emami NK, Jung U, Voy B, Dridi S. Radical Response: Effects of Heat StressInduced Oxidative Stress on Lipid Metabolism in the Avian Liver. Antioxid (Basel) (2020) 10(1):35. doi: 10.3390/antiox10010035

10. Sawka MN, Leon LR, Montain SJ, Sonna LA. Integrated Physiological Mechanisms of Exercise Performance, Adaptation, and Maladaptation to Heat Stress. Compr Physiol (2011) 1(4):1883-928. doi: 10.1002/cphy.c100082

11. Zhai R, Dong X, Feng L, Li S, Hu Z. The Effect of Heat Stress on Autophagy and Apoptosis of Rumen, Abomasum, Duodenum, Liver and Kidney Cells in Calves. Anim (Basel) (2019) 9(10):854. doi: 10.3390/ani9100854

12. Srikandakumar A, Johnson EH, Mahgoub O. Effect of Heat Stress on Respiratory Rate, Rectal Temperature and Blood Chemistry in Omani and Australian Merino Sheep. Small Ruminant Res (2003) 49(2):193-8. doi: 10.1016/s0921-4488(03)00097-x

13. Cui Y, Hao Y, Li J, Bao W, Li G, Gao Y, et al. Chronic Heat Stress Induces Immune Response, Oxidative Stress Response, and Apoptosis of Finishing Pig Liver: A Proteomic Approach. Int J Mol Sci (2016) 17(5):393. doi: 10.3390/ ijms17050393

14. Aengwanich W, Simaraks S. Pathology of Heart, Lung, Liver and Kidney in Broilers Under Chronic Heat Stress. Pathology (2004) 26(3):418.

15. Ravanelli N, Barry H, Schlader ZJ, Gagnon D. Impact of Passive Heat Acclimation on Markers of Kidney Function During Heat Stress. Exp Physiol (2021) 106(1):269-81. doi: 10.1113/EP088637

16. Roncal-Jimenez CA, Sato Y, Milagres T, Andres Hernando A, Garcia G, Bjornstad P, et al. Experimental Heat Stress Nephropathy and Liver Injury Are Improved by Allopurinol. Am J Physiol Renal Physiol (2018) 315(3):F726-33. doi: 10.1152/ajprenal.00543.2017

17. Backhed F, Ley RE, Sonnenburg JL, Peterson DA, Gordon JI. Host-Bacterial Mutualism in the Human Intestine. Science (2005) 307(5717):1915-20. doi: $10.1126 /$ science. 1104816

18. Hill DA, Artis D. Intestinal Bacteria and the Regulation of Immune Cell Homeostasis. Annu Rev Immunol (2009) 28(1):623-87. doi: 10.1146/annurevimmunol-030409-101330

19. Ley RE, Hamady M, Lozupone C, Turnbaugh PJ, Ramey RR, Bircher JS, et al. Evolution of Mammals and Their Gut Microbes. Science (2008) 320 (5883):1647-51. doi: 10.1126/science.1155725

20. Okumura R, Takeda K. Maintenance of Intestinal Homeostasis by Mucosal Barriers. Inflammation Regener (2018) 38(1):5. doi: 10.1186/s41232-018-0063-z

21. Ferrarelli LK. Gut Microbes Help Turn Up the Heat? Sci Signaling (2017) 10 (490). doi: 10.1126/scisignal.aao4947

22. McKenzie C, Tan J, Macia L, Mackay CR. The Nutrition-Gut MicrobiomePhysiology Axis and Allergic Diseases. Immunol Rev (2017) 278(1):277-95. doi: 10.1111/imr.12556

23. SuX, Yin X, Liu Y, Yan X, Zhang S, Wang X, et al. Gut Dysbiosis Contributes to the Imbalance of Treg and Th17 Cells in Graves' Disease Patients by Propionic Acid. J Clin Endocrinol Metab (2020) 105(11):3526-47. doi: 10.1210/dlinem/dgaa511

24. Kasubuchi M, Hasegawa S, Hiramatsu T, Ichimura A, Kimura I. Dietary Gut Microbial Metabolites, Short-Chain Fatty Acids, and Host Metabolic Regulation. Nutrients (2015) 7(4):2839-49. doi: 10.3390/nu7042839

25. Pluznick JL. Microbial Short-Chain Fatty Acids and Blood Pressure Regulation. Curr Hypertens Rep (2017) 19(4):25. doi: 10.1007/s11906-017-0722-5

26. Yao Y, Cai X, Fei W, Ye Y, Zhao M, Zheng C. The Role of Short-Chain Fatty Acids in Immunity, Inflammation and Metabolism. Crit Rev Food Sci Nutr (2020) 10:277. doi: 10.1080/10408398.2020.1854675

27. den Besten G, van Eunen K, Groen AK, Venema K, Reijngoud DJ, Bakker BM. The Role of Short-Chain Fatty Acids in the Interplay Between Diet, Gut
Microbiota, and Host Energy Metabolism. J Lipid Res (2013) 54(9):2325-40. doi: 10.1194/jlr.R036012

28. Wei Y, Liang J, Su Y, Wang J, Amakye WK, Pan J, et al. The Associations of the Gut Microbiome Composition and Short-Chain Fatty Acid Concentrations With Body Fat Distribution in Children. Clin Nutr (2021) 40(5):3379-90. doi: 10.1016/j.clnu.2020.11.014

29. van de Wouw M, Boehme M, Lyte JM, Wiley N, Strain C, O'Sullivan O, et al. Short-Chain Fatty Acids: Microbial Metabolites That Alleviate Stress-Induced Brain-Gut Axis Alterations. J Physiol (2018) 596(20):4923-44. doi: 10.1113/ JP276431

30. Burokas A, Arboleya S, Moloney RD, Peterson VL, Murphy K, Clarke G, et al. Targeting the Microbiota-Gut-Brain Axis: Prebiotics Have Anxiolytic and Antidepressant-Like Effects and Reverse the Impact of Chronic Stress in Mice. Biol Psychiatry (2017) 82(7):472-87. doi: 10.1016/j.biopsych.2016.12.031

31. Lan A, Andriamihaja M, Blouin JM, Liu X, Descatoire V, Desclee de Maredsous C, et al. High-Protein Diet Differently Modifies Intestinal Goblet Cell Characteristics and Mucosal Cytokine Expression in Ileum and Colon. J Nutr Biochem (2015) 26(1):91-8. doi: 10.1016/j.jnutbio.2014.09.007

32. Caporaso JG, Kuczynski J, Stombaugh J, Bittinger K, Bushman FD, Costello EK, et al. QIIME Allows Analysis of High-Throughput Community Sequencing Data. Nat Methods (2010) 7(5):335-6. doi: 10.1038/nmeth.f.303

33. Edgar RC. Search and Clustering Orders of Magnitude Faster Than BLAST. Bioinformatics (2010) 26(19):2460-1. doi: 10.1093/bioinformatics/btq461

34. Grice EA, Kong HH, Conlan S, Deming CB, Davis J, Young AC, et al. Topographical and Temporal Diversity of the Human Skin Microbiome. Science (2009) 324(5931):1190-2. doi: 10.1126/science.1171700

35. Parks DH, Tyson GW, Hugenholtz P, Beiko RG. STAMP: Statistical Analysis of Taxonomic and Functional Profiles. Bioinformatics (2014) 30(21):3123-4. doi: 10.1093/bioinformatics/btu494

36. Huhe X, Hou F, Wu Y, Cheng Y. Bacterial and Fungal Community Structures in Loess Plateau Grasslands With Different Grazing Intensities. Front Microbiol (2017) 8:606. doi: 10.3389/fmicb.2017.00606

37. Li B, Dewey CN. RSEM: Accurate Transcript Quantification From RNA-Seq Data With or Without a Reference Genome. BMC Bioinf (2011) 12(1):323. doi: 10.1186/1471-2105-12-323

38. Hoffman JL. Heat-Related Illness in Children. Clin Pediatr Emergency Med (2001) 2(3):203-10. doi: 10.1016/S1522-8401(01)90006-0

39. Amengual A, Homar V, Romero R, Brooks HE, Ramis C, Gordaliza M, et al. Projections of Heat Waves With High Impact on Human Health in Europe. Global Planetary Change (2014) 119(4):71-84. doi: 10.1016/j.gloplacha. 2014.05.006

40. Nerbass FB, Pecoits-Filho R, Clark WF, Sontrop JM, McIntyre CW, Moist L. Occupational Heat Stress and Kidney Health: From Farms to Factories. Kidney Int Rep (2017) 2(6):998-1008. doi: 10.1016/j.ekir.2017.08.012

41. Gonzalez-Rivas PA, Chauhan SS, Ha M, Fegan N, Dunshea FR, Warner RD. Effects of Heat Stress on Animal Physiology, Metabolism, and Meat Quality: A Review. Meat Sci (2020) 162:108025. doi: 10.1016/j.meatsci. 2019.108025

42. Vandana GD, Sejian V, Lees AM, Pragna P, Silpa MV, Maloney SK. Heat Stress and Poultry Production: Impact and Amelioration. Int J Biometeorol (2021) 65(2):163-79. doi: 10.1007/s00484-020-02023-7

43. Roncal-Jimenez C, Lanaspa MA, Jensen T, Sanchez-Lozada LG, Johnson RJ Mechanisms by Which Dehydration May Lead to Chronic Kidney Disease. Ann Nutr Metab (2015) 66 Suppl 3(Suppl. 3):10-3. doi: 10.1159/000381239

44. Liu Q, Yu Z, Tian F, Zhao J, Zhang H, Zhai Q, et al. Surface Components and Metabolites of Probiotics for Regulation of Intestinal Epithelial Barrier. Microb Cell Fact (2020) 19(1):23. doi: 10.1186/s12934-020-1289-4

45. Zhou X, Qi W, Hong T, Xiong T, Gong D, Xie M, et al. Exopolysaccharides From Lactobacillus Plantarum NCU116 Regulate Intestinal Barrier Function via STAT3 Signaling Pathway. J Agric Food Chem (2018) 66(37):9719-27. doi: $10.1021 /$ acs.jafc. 8 b03340

46. Arnal ME, Zhang J, Messori S, Bosi P, Smidt H, Lalles JP. Early Changes in Microbial Colonization Selectively Modulate Intestinal Enzymes, But Not Inducible Heat Shock Proteins in Young Adult Swine. PloS One (2014) 9(2): e87967. doi: 10.1371/journal.pone.0087967

47. Geddes K, Philpott DJ. A New Role for Intestinal Alkaline Phosphatase in Gut Barrier Maintenance. Gastroenterology (2008) 135(1):8-12. doi: 10.1053/ j.gastro.2008.06.006 
48. Bates JM, Akerlund J, Mittge E, Guillemin K. Intestinal Alkaline Phosphatase Detoxifies Lipopolysaccharide and Prevents Inflammation in Zebrafish in Response to the Gut Microbiota. Cell Host Microbe (2007) 2(6):371-82. doi: 10.1016/j.chom.2007.10.010

49. Malo MS, Alam SN, Mostafa G, Zeller SJ, Johnson PV, Mohammad N, et al. Intestinal Alkaline Phosphatase Preserves the Normal Homeostasis of Gut Microbiota. Gut (2010) 59(11):1476-84. doi: 10.1136/gut.2010.211706

50. Lallès J-P. Microbiota-Host Interplay at the Gut Epithelial Level, Health and Nutrition. J Anim Sci Biotechnol (2016) 7(1):1-8. doi: 10.1186/s40104-0160123-7

51. Arnal ME, Zhang J, Erridge C, Smidt H, Lalles JP. Maternal AntibioticInduced Early Changes in Microbial Colonization Selectively Modulate Colonic Permeability and Inducible Heat Shock Proteins, and Digesta Concentrations of Alkaline Phosphatase and TLR-Stimulants in Swine Offspring. PloS One (2015) 10(2):e0118092. doi: 10.1371/journal. pone.0118092

52. Xue M, Ji X, Liang H, Liu Y, Wang B, Sun L, et al. The Effect of Fucoidan on Intestinal Flora and Intestinal Barrier Function in Rats With Breast Cancer. Food Funct (2018) 9(2):1214-23. doi: 10.1039/c7fo01677h

53. Guo Y, Li H, Liu Z, Li C, Chen Y, Jiang C, et al. Impaired Intestinal Barrier Function in a Mouse Model of Hyperuricemia. Mol Med Rep (2019) 20 (4):3292-300. doi: 10.3892/mmr.2019.10586

54. Sodhi CP, Neal MD, Siggers R, Sho S, Ma C, Branca MF, et al. Intestinal Epithelial Toll-Like Receptor 4 Regulates Goblet Cell Development and Is Required for Necrotizing Enterocolitis in Mice. Gastroenterology (2012) 143 (3):708-718 e5. doi: 10.1053/j.gastro.2012.05.053

55. Gipson IK. Goblet Cells of the Conjunctiva: A Review of Recent Findings. Prog Retin Eye Res (2016) 54:49-63. doi: 10.1016/j.preteyeres.2016.04.005

56. Deckers J, Branco Madeira F, Hammad H. Innate Immune Cells in Asthma. Trends Immunol (2013) 34(11):540-7. doi: 10.1016/j.it.2013.08.004

57. Hooper LV. Bacterial Contributions to Mammalian Gut Development. Trends Microbiol (2004) 12(3):129-34. doi: 10.1016/j.tim.2004.01.001

58. Hooper LV, Gordon JI. Commensal Host-Bacterial Relationships in the Gut. Science (2001) 292(5519):1115-8. doi: 10.1126/science.1058709

59. Goncalves P, Araujo JR, Di Santo JP. A Cross-Talk Between MicrobiotaDerived Short-Chain Fatty Acids and the Host Mucosal Immune System Regulates Intestinal Homeostasis and Inflammatory Bowel Disease. Inflammation Bowel Dis (2018) 24(3):558-72. doi: 10.1093/ibd/izx029

60. Yamada T, Hino S, Iijima H, Genda T, Aoki R, Nagata R, et al. Mucin OGlycans Facilitate Symbiosynthesis to Maintain Gut Immune Homeostasis. EBioMedicine (2019) 48:513-25. doi: 10.1016/j.ebiom.2019.09.008

61. De Rodas B, Youmans BP, Danzeisen JL, Tran H, Johnson TJ. Microbiome Profiling of Commercial Pigs From Farrow to Finish. J Anim Sci (2018) 96 (5):1778-94. doi: 10.1093/jas/sky109
62. Xiao Y, Kong F, Xiang Y, Zhou W, Wang J, Yang H, et al. Comparative Biogeography of the Gut Microbiome Between Jinhua and Landrace Pigs. Sci Rep (2018) 8(1):5985. doi: 10.1038/s41598-018-24289-z

63. Fan P, Liu P, Song P, Chen X, Ma X. Moderate Dietary Protein Restriction Alters the Composition of Gut Microbiota and Improves Ileal Barrier Function in Adult Pig Model. Sci Rep (2017) 7:43412. doi: 10.1038/srep43412

64. Kujawa-Szewieczek A, Adamczak M, Kwiecien K, Dudzicz S, Gazda M, Wiecek A. The Effect of Lactobacillus Plantarum 299v on the Incidence of Clostridium Difficile Infection in High Risk Patients Treated With Antibiotics. Nutrients (2015) 7(12):10179-88. doi: 10.3390/nu7125526

65. Yang WY, Lee Y, Lu H, Chou CH, Wang C. Analysis of Gut Microbiota and the Effect of Lauric Acid Against Necrotic Enteritis in Clostridium Perfringens and Eimeria Side-by-Side Challenge Model. PloS One (2019) 14(5):e0205784. doi: 10.1371/journal.pone.0205784

66. Verhaar BJH, Collard D, Prodan A, Levels JHM, Zwinderman AH, Bäckhed F, et al. Associations Between Gut Microbiota, Faecal Short-Chain Fatty Acids, and Blood Pressure Across Ethnic Groups: The HELIUS Study. Eur Heart (2020) 41(44):4259-67. doi: 10.1093/eurheartj/ehaa704

67. Iwaya C, Nomiyama T, Komatsu S, Kawanami T, Tsutsumi Y, Hamaguchi Y, et al. Exendin-4, a Glucagonlike Peptide-1 Receptor Agonist, Attenuates Breast Cancer Growth by Inhibiting NF-kappaB Activation. Endocrinology (2017) 158(12):4218-32. doi: 10.1210/en.2017-00461

68. Tang ST, Tang HQ, Su H, Wang Y, Zhou Q, Zhang Q, et al. Glucagon-Like Peptide-1 Attenuates Endothelial Barrier Injury in Diabetes via cAMP/PKA Mediated Down-Regulation of MLC Phosphorylation. BioMed Pharmacother (2019) 113(-):108667. doi: 10.1016/j.biopha.2019.108667

Conflict of Interest: The authors declare that the research was conducted in the absence of any commercial or financial relationships that could be construed as a potential conflict of interest.

Publisher's Note: All claims expressed in this article are solely those of the authors and do not necessarily represent those of their affiliated organizations, or those of the publisher, the editors and the reviewers. Any product that may be evaluated in this article, or claim that may be made by its manufacturer, is not guaranteed or endorsed by the publisher.

Copyright (c) $2021 \mathrm{Hu}$, Niu, Chen, Wen, Bao, Mohyuddin, Yong, Liu, Wu, Yu, Ma and Ju. This is an open-access article distributed under the terms of the Creative Commons Attribution License (CC BY). The use, distribution or reproduction in other forums is permitted, provided the original author(s) and the copyright owner(s) are credited and that the original publication in this journal is cited, in accordance with accepted academic practice. No use, distribution or reproduction is permitted which does not comply with these terms. 